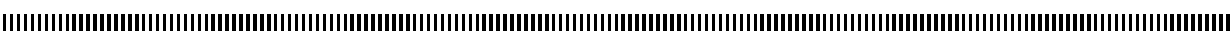
|

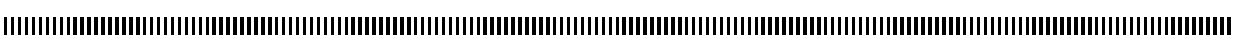
|

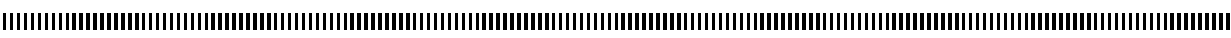
|

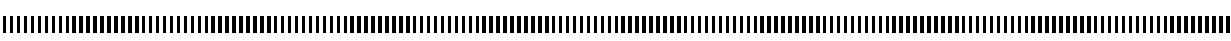

\title{
The peaking phenomenon and singular perturbations
}

\author{
Claude Lobry a - Tewfik Sari a,b,* \\ a Université de Nice et Equipe-Projet INRA-INRIA "Modélisation et Ressources en Eau" \\ UMR Analyse des systèmes et Biométrie. 2 pl. Viala, \\ 34060 Montpellier, FRANCE \\ Claude.Lobry@inria.fr \\ $\mathrm{b}_{\text {Laboratoire de Mathématiques, Informatique et Applications }}$ \\ Université de Haute Alsace, 6 rue des Frères Lumière, \\ 68093 Mulhouse, FRANCE \\ En délégation à l'INRIA Sophia Antipolis - Méditerranée, \\ Equipe-Projet INRA-INRIA "Modélisation et Ressources en Eau” \\ * Coprresponding author : Tewfik.Sari@uha.fr
}

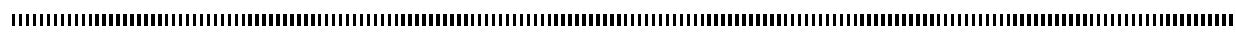
ABSTRACT. We study the asymptotic behaviour, when the parameter $\varepsilon$ tends to 0 , of a class of singularly perturbed triangular systems $\dot{x}=f(x, y), \dot{y}=G(y, \varepsilon)$. We assume that all solutions of the second equation tend to zero arbitrarily fast when $\varepsilon$ tends to 0 . We assume that the origin of equation $\dot{x}=f(x, 0)$ is globally asymptotically stable. Some states of the second equation may peak to very large values, before they rapidly decay to zero. Such peaking states can destabilize the first equation. The paper introduces the concept of instantaneous stability, to measure the fast decay to zero of the solutions of the second equation, and the concept of uniform infinitesimal boundedness to measure the effects of peaking on the first equation. Whe show that all the solutions of the triangular system tend to zero when $\varepsilon \rightarrow 0$ and $t \rightarrow+\infty$. Our results are formulated in both classical mathematics and nonstandard analysis.

RÉSUMÉ. On étudie le comportement asymptotique, lorsque le paramètre $\varepsilon$ tend vers 0 , de systèmes triangulaires singulierement perturbés de la forme $\dot{x}=f(x, y), \dot{y}=G(y, \varepsilon)$. On suppose que toutes les solutions de la deuxième équation tendent vers zéro arbitrairement rapidement quand $\varepsilon$ tend vers 0 . On suppose que le système $\dot{x}=f(x, 0)$ admet l'origine comme équilibre globalement asymptotiquement stable. Certaines solutions de la deuxième équation peuvent présenter un transitoire avec un pic très grand avant de décroître rapidement vers zéro. C'est ce phénomène de peaking qui peut destabiliser la première équation. On introduit le concept de stabilité instantanée, pour mesurer la décroissance rapide vers zéro des solutions de la deuxième équation, et le concept de système uniformément infinitésimalement borné pour mesurer les effets du peaking sur la première équation. On montre que les solutions du système triangulaire tendent vers zéro quand $\varepsilon \rightarrow 0$ et $t \rightarrow+\infty$. Nos résultats sont formulés dans le cadre de l'analyse non standard et sont traduits en termes classiques.

KEYWORDS : peaking phenomenon, asymptotic stability, singular perturbations, nonstandard analysis

MOTS-CLÉS : phénomène du peaking, stabilité asymptotique, perturbations singulières, analyse non standard

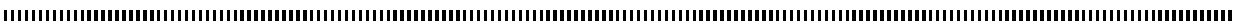




\section{Introduction}

In this paper, we are concerned with the asymptotic behavior, when the parameter $\varepsilon$ tends to 0 , of nonlinear triangular systems

$$
\dot{x}=f(x, y), \quad \dot{y}=G(y, \varepsilon)
$$

where ${ }^{\cdot}=d / d t, x \in \mathbb{R}^{n}, y \in \mathbb{R}^{m}, \varepsilon>0$ and the mappings

$$
\left.f: \mathbb{R}^{n} \times \mathbb{R}^{m} \rightarrow \mathbb{R}^{n} \text { and } G: \mathbb{R}^{m} \times\right] 0, \varepsilon_{0}\left[\rightarrow \mathbb{R}^{m}\right.
$$

are of class $\mathcal{C}^{1}$. Notice that the limit of $G$ is not assumed to be defined when $\varepsilon \rightarrow 0$, so that system (1) is a singular perturbation. We can think of the first equation in system (1) as a controlled system taking its inputs from the second equation in system (1). The zero-input system is the system

$$
\dot{x}=f(x, 0) \text {. }
$$

We assume that

H1 : system (2) has 0 as a globally asymptotically stable (GAS) equilibrium.

The condition H1 implies that the solutions of (2) tend to 0 as $t \rightarrow+\infty$. Our aim is to state conditions on $f$ and $G$ such that the solutions of system (1) tend to zero when $\varepsilon \rightarrow 0$ and $t \rightarrow+\infty$. Intuitively, if we require that

$$
\text { the solutions of } \dot{y}=G(y, \varepsilon) \text { tend to } 0 \text { arbitrarily fast in } t \text { when } \varepsilon \rightarrow 0 \text {, }
$$

then, the idea that the solutions of system (1) ought to tend to zero appears plausible for the following heuristics : Since (3) holds, the second equation in system (1) drives any initial condition very fast in $t$ near the manifold $y=0$, where the zero-input system takes over and drives $x$ to zero. Due to the peaking phenomenon, this idea fails. Of course, some solutions of the second equation in system (1) may peak to very large values before they decay to zero. The interaction of this peaking with the nonlinear growth in the first equation in system (1) could destabilize system (1). In general the origin of system (1) is not GAS, even if there is no peaking. The best result one can expect for system (1) is that its origin is Practically Semi Globally Asymptotically Stable (PSGAS) when $\varepsilon \rightarrow 0$ (see Definition 5.2).

Our first objective is to give a precise meaning to the sentence (3). For this purpose we shall use the Nonstandard Analysis (NSA) of Abraham Robinson [26]. The idea of using NSA in perturbation theory of differential equations goes back to the seventies with the Reebian school (see Section E). To have an idea of the rich literature on the subject the reader is referred to $[2,7,10,20,21,23,24,30]$.

In NSA, instead of considering a family of systems (1) depending on the parameter $\varepsilon$ and dealing with its asymptotic propreties when $\varepsilon \rightarrow 0$, we consider just one (nonstandard) system

$$
\dot{x}=f(x, y), \quad \dot{y}=g(y),
$$

where $g(y)$ plays the role of $G(y, \varepsilon)$ with $\varepsilon$ a fixed positive infinitesimal real number. We look to the (external) properties of system (4). 
In this paper, we introduce the concepts of instantaneous stability, which quantify the fast decay to zero of the solutions of the second equation in system (4) as well as the concepts of uniform infinitesimal boundedness which measure the effects of the solutions of the second equation in system (4) on the first equation in this system. All these concepts are defined in both standard and nonstandard terms. Also, we introduce the concept of steadiness which measure the effects of a peaking control on a system.

Automatic control engineers like to stabilize plants as fast as possible. If the system is linear one tries to put the eigenvalues as far as possible in the left of the complex plane but it is well known that this may be dangerous in the presence of nonlinear, even small, perturbations. Far from being stabilized the system can explode to infinity. In 1988, M. Canalis and P. Yalo [6] remarked the paper [15] of P.V. Kokotovic and R. Marino which for the first time (to our knowledge) payed attention to this problem. They published a short paper which clarifies one of the thorough reasons of this phenomenon : Before it goes to zero a trajectory makes an excursion in the "neighborhood of infinity". Independently H.J. Sussmann and P.V. Kokotovic popularized this phenomenon under the name "peaking phenomenon" in a paper [29] which, moreover, gives some conditions to secure oneself against it. The notion of instantaneous stabilizabilty was previously defined (see [6]) for linear systems within the NSA framework. Our concept of instantaneous stability for a general system is a straightforward extension of their definition.

The paper is organized as follows. In Section 2 we recall Tikhonov's theorem that describes the case where $G$ is of the form $G(y, \varepsilon)=\frac{1}{\varepsilon} G(y)$. In Section 3 we consider the linear case, where $G$ is of the form $G(y, \varepsilon)=G(\varepsilon) y$ and we explain why the peaking phenomenon can destabilize the first equation. In Section 4, we give our main (nonstandard) result (Theorems 4.7) which extends Tikhonov's theorem and we deduce from this theorem two results (Theorems 4.8 and 4.13) on the global asymptotic stability of the origin of system (4). In Section 5, we provide the standard formulations (Theorems 5.6 and 5.9) of these stability results in terms of Practical Semi Global Asymptotic Stability. Typical examples of applications of these results are given in Section 6. In Section 7, we give the definition of a peaking of order $\alpha$ for a general nonlinear system and investigate the particular case of linear systems in the Luenberger-Brunowsky canonical form. In Section 8 , we motivate the case where the mapping $g$ in (4) depends also on $x$. This case arises in control problems where the feedback is computed, not on the state, but on an estimation of the state given by an observer. In Appendix A, we give a short description of Internal Set Theory (IST) which is an axiomatic approach, given by Nelson [25] of NSA. In Appendix B, we give some useful results of nonstandard asymptotic theory. In Appendix C, we prove the equivalence between the standard and the nonstandard definitions of the concepts introduced in Sections 4 and 5. In Appendix D, we give the proofs of Theorems 2.3, 5.6 and 5.9. In Appendix E we give some informations on NSA and differential equations.

Theorems 2.3, 5.6 and 5.9 are formulated in classical mathematics, but their proofs need IST. We don't give classical proofs because IST is a conservative extension of ordinary mathematics, that is, every classical statement which is proved in IST is a theorem of ordinary mathematics. We prefer the IST language in this kind of study because the nonstandard statements are more appealing for us in view of applications. Nonetheless, it's a matter of taste. 
A control $u$ is a function $t \rightarrow u(t)$ defined for $0 \leq t<\infty$ which takes values on $\mathbb{R}^{m}$ and is bounded and mesurable. A control system $f$ is a system

$$
\dot{x}=f(x, u), \quad x \in \mathbb{R}^{n}, u \in \mathbb{R}^{m},
$$

such that the map $f: \mathbb{R}^{n} \times \mathbb{R}^{m} \rightarrow \mathbb{R}^{n}$ is of class $\mathcal{C}^{1}$. If $u$ is a control, we use $x^{f}\left(t, t_{0}, x_{0}, u(\cdot)\right)$ to denote the solution of

$$
\dot{x}=f(x, u(t)), \quad x\left(t_{0}\right)=x_{0} .
$$

When $t_{0}=0$, we denote the solution simply by $x^{f}\left(t, x_{0}, u(\cdot)\right)$. When there is no risk of confusion, $f, t_{0}, x_{0}$ or $u$ could be omitted. Let $(x(t), y(t))$ be the solution of system (1) with initial condition $x(0)=x_{0}, y(0)=y_{0}$. According to our notations, we have

$$
x^{(f, G)}\left(t,\left(x_{0}, y_{0}\right), \varepsilon\right)=x^{f}\left(t, x_{0}, y(\cdot)\right) \text { where } y(t)=y^{G}\left(t, y_{0}, \varepsilon\right) .
$$

Throughout the paper, we use the following abbreviations : 'GAS' is read 'Global Asymptotic Stability' (or 'Globally Asymptotically Stable'), 'IS' is read 'Instantaneous Stability' (or 'Instantaneously Stable'), 'UIB' is read 'Uniform Infinitesimal Boundedness' (or 'Uniformly Infinitesimally Bounded'), ' $\omega$-IS' is read ' $\omega$-Instantaneous Stability' (or ' $\omega$-Instantaneously Stable'), ' $\omega$-UIB' is read ' $\omega$-Uniform Infinitesimal Boundedness' (or ' $\omega$-Uniformly Infinitesimally Bounded').

\section{Tikhonov's theorem}

The case where system (1) is of the form

$$
\dot{x}=f(x, y), \quad \varepsilon \dot{y}=G(y),
$$

with $G: \mathbb{R}^{m} \rightarrow \mathbb{R}^{m}$ of class $\mathcal{C}^{1}$, is a particular case of slow and fast systems considered by Tikhonov's theory. $[13,14,20]$. We assume that

$\mathrm{H} 2$ : the vector field $y \mapsto G(y)$ has 0 as a GAS equilibrium.

Theorem 2.1 (Tikhonov) Assume that $f$ and $G$ are standard, $\varepsilon$ is infinitesimal positive and $H 2$ holds. Then for all limited $x_{0}$ and $y_{0}$, the $x$-component $x(t)$ of the solution of system (6) with initial condition $\left(x_{0}, y_{0}\right)$ satisfies $x(t) \simeq x_{0}(t)$ as long as $t$ and $x_{0}(t)$ are limited, where $x_{0}(t)$ is the solution of

$$
\dot{x}=f(x, 0), \quad x(0)=x_{0} .
$$

Moreover, if $H 1$ also holds then $x(t) \simeq x_{0}(t)$ for all $t \geq 0$.

Proof. Define $\xi(\tau)=x(\varepsilon \tau)$ and $\eta(\tau)=y(\varepsilon \tau)$, where $(x(t), y(t))$ is the solution of (6) with initial condition $\left(x_{0}, y_{0}\right)$. System (6) becomes

$$
\xi^{\prime}=\varepsilon f(\xi, \eta), \quad \eta^{\prime}=G(\eta)
$$

where $^{\prime}=d / d \tau$. Since $f$ is limited for all $(\xi, \eta)$ limited and $\varepsilon$ is infinitesimal positive, this system is a regular perturbation of system

$$
\xi^{\prime}=0, \quad \eta^{\prime}=G(\eta) .
$$


According to the Short Shadow Lemma (Lemma B.1), for all limited initial condition $\left(x_{0}, y_{0}\right)$ we have $\xi(\tau) \simeq x_{0}$ as long as $\tau$ is limited. By Robinson's Lemma (Lemma A.2), there exists an unlimited $s$ such that $\xi(\tau) \simeq x_{0}$ for all $\tau \in[0, s]$. we can chose $s$ such that $t^{*}=\varepsilon s$ is infinitesimal. By H2 (and Proposition 4.3), we have $\eta(\tau) \simeq 0$ for all $\tau \simeq+\infty$, so that $y(t)=\eta(t / \varepsilon) \simeq 0$ for all $t \geq t^{*}$. Let $x_{0}^{*}=x\left(t^{*}\right)$. By Proposition B.4, we have $x(t) \simeq x^{f}\left(t, x_{0}^{*}, 0\right)$ as long as $t$ and $x^{f}\left(t, x_{0}^{*}, 0\right)$ are limited. Since $x_{0}^{*} \simeq x_{0}$, again by the Short Shadow Lemma, we have $x^{f}\left(t, x_{0}^{*}, 0\right) \simeq x^{f}\left(t, x_{0}, 0\right)=: x_{0}(t)$ as long as $t$ and $x_{0}(t)$ are limited. This proves the first part of the theorem. Assume now that assumption H1 holds. Then $x_{0}(t)$ is limited for all $t \geq 0$ and $x_{0}(t) \simeq 0$ for all $t \simeq+\infty$. Thus, $x(t) \simeq x_{0}(t)$ for all limited positive $t$. By Robinson's Lemma, there exists $t_{1}$ unlimited such that $x(t) \simeq x_{0}(t)$ for all $t \in\left[0, t_{1}\right]$. Thus, we have $x(t) \simeq x_{0}(t) \simeq 0$ for all unlimited $t \leq t_{1}$. By $\mathrm{H} 2$, we have $y(t) \simeq 0$ for all $t \geq t_{1}$. By Proposition B.5, we have $x(t) \simeq 0$ for all $t \geq t_{1}$. Hence $x(t) \simeq x_{0}(t)$ for all $t \geq 0$.

Theorem 2.2 Assume that $f$ and $G$ are standard, $\varepsilon$ is infinitesimal positive, and $H 1$ and $H 2$ hold. Then for all limited $x_{0}$ and $y_{0}$, the solution $(x(t), y(t))$ of $(6)$ with initial condition $\left(x_{0}, y_{0}\right)$ satisfies $x(t) \simeq 0$ and $y(t) \simeq 0$ for all positive unlimited $t$.

Proof. Let $x_{0}$ and $y_{0}$ be limited. Let $(x(t), y(t))$ be the solution of (6) with initial condition $\left(x_{0}, y_{0}\right)$. By Theorem 2.1, we have $x(t) \simeq x_{0}(t)$ for all $t \geq 0$. By H1, we have $x_{0}(t) \simeq 0$ for all $t \simeq+\infty$. By H2, we have $y(\varepsilon \tau) \simeq 0$ for all $\tau \simeq+\infty$. Hence $x(t) \simeq 0$ and $y(t) \simeq 0$ for all $t \simeq+\infty$.

Theorem 2.3 Assume that H1 and H2 hold. For all $\left(x_{0}, y_{0}\right)$, the solution $(x(t, \varepsilon), y(t, \varepsilon))$ of (6) with initial condition $\left(x_{0}, y_{0}\right)$ tends to 0 as $t \rightarrow+\infty$ and $\varepsilon \rightarrow 0$. For every $A>0$, the convergence is uniform with respect to $x_{0}$ and $y_{0}$ for $\left\|x_{0}\right\| \leq A$ and $\left\|y_{0}\right\| \leq A$.

The proof is given in Appendix D.

Example. Consider the system

$$
\dot{x}=-x+x^{2} y, \quad \varepsilon \dot{y}=-y .
$$

Conditions $\mathrm{H} 1$ and $\mathrm{H} 2$ are satisfied. By Theorem 2.3 the solutions of (7) tend to 0 as $\varepsilon \rightarrow 0$ and $t \rightarrow+\infty$. Since

$$
\frac{d}{d t}(x y)=x y(x y-1-1 / \varepsilon)
$$

we see that the hyperbola $x y=1+1 / \varepsilon$ consists of two trajectories. Thus the origin of (7) is not GAS. Tikhonov's theorem asserts that the $x$-component of the solution $x(t, \varepsilon)$ of (7) with initial condition $\left(x_{0}, y_{0}\right)$ is such that, for all $t \geq 0$

$$
\lim _{\varepsilon \rightarrow 0} x(t, \varepsilon)=x_{0}(t):=x_{0} \mathrm{e}^{-t}
$$

where $x_{0}(t)$ is the solution of the zero-input system

$$
\dot{x}=-x, \quad x(0)=x_{0} .
$$

By explicit solutions, it is easy to show that the basin of attraction of the origin of (7) is the set $\{x y<1+1 / \varepsilon\}$. 


\section{The Peaking phenomenon}

Let us consider now the particular case where system (1) is of the form

$$
\dot{x}=f(x, y), \quad \dot{y}=G(\varepsilon) y,
$$

where $G(\varepsilon)$ is a square matrix of order $m$. We assume that :

$\mathrm{H} 3:$ the real parts of the eigenvalues of $G(\varepsilon)$ tend to $-\infty$ when $\varepsilon \rightarrow 0$.

System (9) was considered in automatic control literature. In this context the second equation in system (9) is understood as a "high gain" dynamic feedback [29]. More precisely, we start with a state feedback-partially linear system of the form

$$
\dot{x}=f(x, y), \quad \dot{y}=A y+B u,
$$

where the pair $(A, B)$ is controllable. The second equation can be easily stabilized by $u=K y$, where $K$ is designed such that the matrix $G:=A+B K$ is Hurwitz. Asymptotic stability of the origin of the full closed-loop system

$$
\dot{x}=f(x, y) \quad \dot{y}=G y
$$

will now folow from assumption $\mathrm{H} 1$ and the concept of input to state stability (see [14], p. 548). What about global stabilization ? If the linear feedback control $u=K y$ is designed to assign the eigenvalues of $G$ far to the left in the complex plane so that the solutions of $\dot{y}=G y$ decay to zero arbitrarily fast, one might think that the origin of the triangular system (10) can be GAS. It turns out that such a strategy may fail because of the so called "peaking phenomenon". The following example of Sussmann and Kokotovic (cf. [29] Example 1.1) shows a system of type (9) such that the solution $y(t, \varepsilon)$ becomes unbounded when $\varepsilon \rightarrow 0$, even if its decay to zero is arbitrary fast. In that case, the origin of (9) is not GAS. Even worse, for some initial conditions, the solution escapes to infinity in finite time.

Example. Consider the three dimensional system

$$
\dot{x}=\left(1+y_{2}\right) \varphi(x), \quad \dot{y}=G(\varepsilon) y,
$$

where $\varphi(x)=-x^{3} / 2$ and

$$
G(\varepsilon)=\left(\begin{array}{cc}
0 & 1 \\
-1 / \varepsilon^{2} & -2 / \varepsilon
\end{array}\right)
$$

Condition H1 is satisfied. Since both eigenvalues of $G(\varepsilon)$ are equal to $-1 / \varepsilon$, condition $\mathrm{H} 3$ is also satisfied. The exponential matrix

$$
\mathrm{e}^{t G(\varepsilon)}=\left(\begin{array}{cc}
1+t / \varepsilon & t \\
-t / \varepsilon^{2} & 1-t / \varepsilon
\end{array}\right) \mathrm{e}^{-t / \varepsilon}
$$

shows that as $\varepsilon \rightarrow 0$, the solution $y(t, \varepsilon)=\mathrm{e}^{t G(\varepsilon)} y_{0}$ will decay to zero arbitrarily fast. The component $\left(t / \varepsilon^{2}\right) \mathrm{e}^{-t / \varepsilon}$ of (12) reaches the value $1 /(\varepsilon \mathrm{e})$ at $t=\varepsilon$. Then some solutions have a transient behaviour with a peak of order $1 / \varepsilon$ before they rapidly decay to 0 . This phenomenon is known as the peaking phenomenon. The interaction of this peaking with 
the nonlinear growth in the first equation in system (11) could destabilize the system. Let $y_{0}=\left(y_{10}, y_{20}\right)$, then we have

$$
x(t, \varepsilon)=\frac{x_{0}}{\sqrt{1+x_{0}^{2}\left[t-y_{10}+\left(y_{10}(1+t / \varepsilon+1)+y_{20} t\right) \mathrm{e}^{-t / \varepsilon}\right]}} .
$$

If $x_{0}^{2} y_{10}>1$ the solution will have a finite escape time $t_{e}(\varepsilon)>0$ and $t_{e}(\varepsilon)$ tends to zero when $\varepsilon \rightarrow 0$. For $\varepsilon$ small enough, the solutions are attracted to 0 as soon as $x_{0}^{2} y_{10}<1$ and for all $t>0$ we have

$$
\lim _{\varepsilon \rightarrow 0} x(t, \varepsilon)=x_{0}(t):=\frac{x_{0}^{*}}{\sqrt{1+x_{0}^{* 2} t}} .
$$

Here, $x_{0}(t)$ is the solution of the zero-input system

$$
\dot{x}=-x^{3} / 2, \quad x(0)=x_{0}^{*}:=\frac{x_{0}}{\sqrt{1-x_{0}^{2} y_{10}}} .
$$

Since the limit (13) does not hold for $t=0$, there is a boundary layer at $t=0$ which quickly drives the state $x(t, \varepsilon)$ from $x_{0}$ to $x_{0}^{*}$. The peaking phenomenon explains both this boundary layer for the solutions attracted to 0 and the destabilizing effect of the second equation in system (11) on the first equation in this system.

Example. If $\varphi(x)=-x$ in (11) then the origin is GAS in spite of peaking. However, the solutions still have a boundary layer at $t=0$. Since

$$
x(t, \varepsilon)=x_{0} \exp \left(y_{10}-t-\left[y_{10}(1+t / \varepsilon)+y_{20} t\right] \mathrm{e}^{-t / \varepsilon}\right)
$$

we see that, all the solutions are attracted to 0 and for all $t>0$ we have

$$
\lim _{\varepsilon \rightarrow 0} x(t, \varepsilon)=x_{0}(t):=x_{0}^{*} \mathrm{e}^{-t} .
$$

Here, $x_{0}(t)$ is the solution of the zero-input system

$$
\dot{x}=-x \quad x(0)=x_{0}^{*}:=x_{0} \mathrm{e}^{y_{10}} .
$$

The boundary layer at $t=0$ quickly drives the state $x(t)$ from $x_{0}$ to $x_{0}^{*}$.

The limit (8) was obtained from Tikhonov's theorem. The limits (13) and (15) were obtained by direct computation, because Tikhonov's theorem does not apply to (11).

\section{Nontandard results}

\subsection{S-Global Asymptotic Stability}

The results in Section 2 show that the origin of (6) cannot be GAS in general. The best result one can expect for system (6) is the one given by Theorem 2.2, which asserts that when $\varepsilon$ is infinitesimal positive, then for every limited initial condition the solution of (6) is infinitesimal, as long as $t \simeq+\infty$. This notion deserves a definition. Consider the (possibly nonstandard) system

$$
\dot{z}=F(z)
$$

where $z \in \mathbb{R}^{p}$ and $F: \mathbb{R}^{p} \rightarrow \mathbb{R}^{p}$. 
Definition 4.1 The origin of (17) is S-GAS if for every limited $z_{0}$ and for every positive unlimited $t$, we have $z^{F}\left(t, z_{0}\right) \simeq 0$.

Remark 4.2 We can reformulate Theorem 2.2 by stating that for all positive infinitesimal $\varepsilon$, the origin of system (6) is S-GAS.

In NSA we use the prefix S to avoid confusion between the standard and the nonstandard corresponding notions. The notion of S-GAS corresponds effectively to the usual GAS and we have :

Proposition 4.3 Assume that $F$ is standard. The origin of (17) is GAS if and only if it is S-GAS.

The proof is given in Appendix A. This property does not hold for a nonstandard system. Indeed, if $\varepsilon$ is infinitesimal positive, then the origin of system $\dot{z}=z(\varepsilon z-1)$ is S-GAS but not GAS.

\subsection{Instantaneous Stability and Uniform Infinitesimal Boundedness}

In the previous particular cases (6) and (9) of system (4), the fast attractivity of the origin was guaranteed by the special form of the second equation in the system and by conditions $\mathrm{H} 2$ or $\mathrm{H} 3$. In the general case, there is no such criteria and we must introduce fast attractivity as a hypothesis. For this purpose, we need the following definitions.

Definition 4.4 A function $t \rightarrow u(t)$ is said to be an impulse if $u(t) \simeq 0$, for all positive non infinitesimal $t$.

Definition 4.5 The origin of system $\dot{y}=g(y)$ is instantaneously stable (IS) if for every limited $y_{0}$, the function $t \rightarrow y^{g}\left(t, y_{0}\right)$ is an impulse.

Example. If $\varepsilon$ is infinitesimal positive, the origin of the second equation in system (11) is IS.

The effects of any possible peaking of the solutions of the second equation in system (4) on the first equation in this system must be controlled. Thus, we need a hypothesis on the behaviour of the $x$-component of the solutions of (4) during the very short time where the peaking can destabilize the system. For this purpose, we need the following definition.

Definition 4.6 The system (4) is uniformly infinitesimally bounded (UIB) if for all limited $x_{0}$ and $y_{0}$ and for all positive infinitesimal $t$, the $x$-component $x(t)=x^{(f, g)}\left(t,\left(x_{0}, y_{0}\right)\right)$ of the solution of (4) with initial condition $\left(x_{0}, y_{0}\right)$ is limited.

We consider the condition below :

H4 : system (4) is UIB and the origin of system $\dot{y}=g(y)$ is IS.

Theorem 4.7 Assume that $f$ is standard and $H 4$ holds. Then for all limited $x_{0}$ and $y_{0}$, there exists a limited $x_{0}^{*}$ such that the $x$-component $x(t)$ of the solution of (4) with initial 
condition $\left(x_{0}, y_{0}\right)$ satisfies $x(t) \simeq x_{0}(t)$, as long as $x_{0}(t)$ is limited and $t$ is appreciable positive, where $x_{0}(t)$ is the solution of the zero-input system (2), with initial condition $x_{0}^{*}$. Moreover, if $H 1$ also holds then $x(t) \simeq x_{0}(t)$ for all non infinitesimal positive $t$.

Proof. We have $x(t)=x^{(f, g)}\left(t,\left(x_{0}, y_{0}\right)\right)=x^{f}\left(t, x_{0}, y(\cdot)\right)$, where $y(t)=y^{g}\left(t, y_{0}\right)$. Let $x_{0}$ and $y_{0}$ be limited. Since the origin of $\dot{y}=g(y)$ is IS, $y(t) \simeq 0$ for every $t \gg 0$. By Lemma A.2, there exists $t^{*} \simeq 0$ such that $y(t) \simeq 0$ for every $t \geq t^{*}$. Since (4) is UIB, $x(t)$ is limited for $0 \leq t \leq t^{*}$. Let $x_{0}^{*}=x\left(t^{*}\right)$. By Proposition B.4, for all $t \geq t^{*}$ we have $x(t) \simeq x^{f}\left(t, x_{0}^{*}, 0\right)=: x_{0}(t)$, as long as $t$ and $x_{0}(t)$ are limited. This proves the first part of the theorem. Assume now that $\mathrm{H} 1$ holds, then $x_{0}(t)$ is limited for all $t \geq 0$ and $x_{0}(t) \simeq 0$ for all unlimited positive $t$. Thus, $x(t) \simeq x_{0}(t)$ for all appreciable positive $t$. By Lemma A.2, there exists $t_{1}$ unlimited such that $x(t) \simeq x_{0}(t)$ for all non infinitesimal positive $t \leq t_{1}$. Thus, we have $x(t) \simeq x_{0}(t) \simeq 0$ for all unlimited $t \leq t_{1}$. By IS, we have $y(t) \simeq 0$ for all $t \gg 0$ and hence for all $t \geq t_{1}$. By Proposition B.5, we have $x(t) \simeq 0$ for all $t \geq t_{1}$. Hence, $x(t) \simeq x_{0}(t)$ for all $t \gg 0$.

This result shows that a solution of system (4) starting from a limited point $\left(x_{0}, y_{0}\right)$ is approximated by a solution of the zero-input system (2) starting from a limited point $x_{0}^{*}$. It is a Tikhonov-like result (compare with Theorem 2.1). In Tikhonov's case we had $x_{0}^{*} \simeq x_{0}$. However in the general case, due to the possible peaking of the solutions of the second equation in system (4), as shown in (14) and (16), $x_{0}^{*} \nsucceq x_{0}$ and there is a boundary layer at $t=0$.

Theorem 4.8 Assume that $f$ is standard, and that $\mathrm{Hl}$ and $\mathrm{H} 5$ hold. Then the origin of system (4) is S-GAS.

Proof. Let $x_{0}$ and $y_{0}$ be limited. Let $(x(t), y(t))$ be the solution of (4) with initial condition $\left(x_{0}, y_{0}\right)$. By Theorem 4.7, we have $x(t) \simeq x_{0}(t)$ for all $t \gg 0$. By H1, we have $x_{0}(t) \simeq 0$ for all $t \simeq+\infty$. By IS, we have $y(t) \simeq 0$ for all $t \gg 0$. Hence, $x(t) \simeq 0$ and $y(t) \simeq 0$ for all $t \simeq+\infty$, that is to say, the origin of (4) is S-GAS.

\section{3. $\omega$-Instantaneous Stability and $\omega$-Uniform Infinitesimal Boundedness}

In the case of the partially linear system (9), the order of magnitude of the velocity with which the solutions tend to 0 was measured in terms of the spectrum of the matrix $G(\varepsilon)$, which is no longer possible in the nonlinear case. In this section, we quantify more precisely the behaviour of the solutions of system (4). For this purpose, we need the following definitions. Let $\omega$ be an unlimited positive real number.

Definition 4.9 A function $t \rightarrow u(t)$ is said to be an $\omega$-impulse if $u(\tau / \omega) \simeq 0$ for all positive unlimited $\tau$.

Definition 4.10 The origin of system $\dot{y}=g(y)$ is $\omega$-IS if for every limited $y_{0}$, the function $t \rightarrow y^{g}\left(t, y_{0}\right)$ is an $\omega$-impulse.

Example. Let $\varepsilon$ be infinitesimal positive. The origin of the second equation in system (11) is not $\omega$-IS where $\omega=1 / \varepsilon$. Indeed, the components of the exponential matrix (12) are infinitesimal as long as $t=\varepsilon \tau$ and $\tau \simeq+\infty$, apart from the component

$$
h(t)=-\left(t / \varepsilon^{2}\right) \mathrm{e}^{-t / \varepsilon},
$$


for which we have $h(-\varepsilon \ln \varepsilon)=\ln \varepsilon$, which is not limited. The origin of the second equation in system (11) is $\omega$-IS with $\omega=-1 /(\varepsilon \ln \varepsilon)$. Indeed, we have $h(\tau / \omega)=$ $\tau \varepsilon^{\tau-1} \ln \varepsilon$, which is infinitesimal for all $\tau \simeq+\infty$.

Definition 4.11 The system (4) is $\omega$-UIB iffor all limited $x_{0}$ and $y_{0}$, there exists a positive unlimited s such that the $x$-component $x(t)=x^{(f, g)}\left(t,\left(x_{0}, y_{0}\right)\right)$ is limited for all $t \in$ $[0, s / \omega]$.

We consider the condition below.

H5 : there exists a positive unlimited real number $\omega$ such that the origin of system $\dot{y}=g(y)$ is $\omega$-IS, and system (4) is $\omega$-UIB.

Theorem 4.12 The conclusion of Theorem 4.7 holds if assumption H4 is replaced by assumption $\mathrm{H} 5$.

Proof. We have $x(t)=x^{(f, g)}\left(t,\left(x_{0}, y_{0}\right)\right)=x^{f}\left(t, x_{0}, y(\cdot)\right)$ where $y(t)=y^{g}\left(t, y_{0}\right)$. Let $x_{0}$ and $y_{0}$ be limited. Since (4) is $\omega$-UIB, there exists $s \simeq+\infty$ such that $x^{f}\left(t, x_{0}, y(\cdot)\right)$ is limited for $0 \leq t \leq s / \omega$. Since $\omega$ is unlimited, we can chose $s$ such that $t^{*}=s / \omega$ is infinitesimal. Since the origin of the second equation in system (4) is $\omega$-IS, we have $y(t) \simeq 0$, for all $t \geq t^{*}$. Let $x_{0}^{*}=x\left(t^{*}\right)$. By Proposition B.4, for all $t \geq t^{*}$ we have $x(t) \simeq x^{f}\left(t, x_{0}^{*}, 0\right)=: x_{0}(t)$, as long as $t$ and $x_{0}(t)$ are limited. This proves the first part of the theorem. Assume now that assumption $\mathrm{H} 1$ holds. Then, $x_{0}(t)$ is limited for all $t \geq 0$ and $x_{0}(t) \simeq 0$ for all unlimited positive $t$. Thus, $x(t) \simeq x_{0}(t)$ for all appreciable positive $t$. By Lemma A.2, there exists $t_{1}$ unlimited such that $x(t) \simeq x_{0}(t)$ for all non infinitesimal positive $t \leq t_{1}$. Thus, we have $x(t) \simeq x_{0}(t) \simeq 0$ for all unlimited $t \leq t_{1}$. By $\omega$-IS, we have $y(\tau / \omega) \simeq 0$ for all $\tau \simeq+\infty$ and hence, $y(t) \simeq 0$ for all $t \geq t_{1}$. By Proposition B.5, we have $x(t) \simeq 0$ for all $t \geq t_{1}$. Hence, $x(t) \simeq x_{0}(t)$ for all $t \gg 0$.

Theorem 4.13 Assume that $f$ is standard and that HI and H5 hold. Then the origin of (4) is S-GAS.

Proof. Let $x_{0}$ and $y_{0}$ be limited. Let $(x(t), y(t))$ be the solution of (4) with initial condition $\left(x_{0}, y_{0}\right)$. By Theorem 4.12, we have $x(t) \simeq x_{0}(t)$ for all $t \gg 0$. By $\mathrm{H} 1$, we have $x_{0}(t) \simeq 0$ for all $t \simeq+\infty$. By $\omega$-IS, we have $y(\tau / \omega)$ for all $\tau \simeq+\infty$. Hence, $x(t) \simeq 0$ and $y(t) \simeq 0$ for all $t \simeq+\infty$. Thus, the origin of (4) is S-GAS.

\section{Standard results}

\subsection{Practical Semi Global Asymptotic Stability}

It is well known (see [12], Theorem 38.1 or [27], Exercise 2.9, p. 9)) that uniform attractivity with respect to the initial condition implies asymptotic stability. Thus we have :

Proposition 5.1 The origin of (17) is GAS if and only if it is uniformly attractive with respect to the initial condition on every ball centered in the origin. 
A nonstandard proof of Proposition 5.1 is given in Appendix A. Hence, the origin of (17) is GAS if and only if for every $A>0, z^{F}\left(t, z_{0}\right) \rightarrow 0$ as $t \rightarrow+\infty$, the convergence being uniform with respect to $z_{0}$ for $\left\|z_{0}\right\| \leq A$. Consider now a system depending on a parameter :

$$
\dot{z}=F(z, \varepsilon)
$$

where $z \in \mathbb{R}^{p}$ and $F: \mathbb{R}^{p} \times \mathbb{R} \rightarrow \mathbb{R}^{p}$.

Definition 5.2 The origin of (18) is Practically Semi Globally Asymptotically Stable (PS$G A S)$ when $\varepsilon \rightarrow 0$ if for every $A>0, z^{F}\left(t, z_{0}, \varepsilon\right) \rightarrow 0$ as $t \rightarrow+\infty$ and $\varepsilon \rightarrow 0$, the convergence being uniform with respect to $z_{0}$ for $\left\|z_{0}\right\| \leq A$.

Remark 5.3 We can formulate Theorem 2.3 by saying that the origin of system (6) is $P S G A S$ when $\varepsilon \rightarrow 0$.

Notice that a point that is PSGAS is not GAS. Indeed, the origin of system

$$
\dot{z}=z(\varepsilon z-1)
$$

is PSGAS when $\varepsilon \rightarrow 0$. However, it is a locally asymptotically stable but not GAS equilibrium. The origin of system

$$
\dot{z}=\varepsilon-z
$$

is PSGAS when $\varepsilon \rightarrow 0$. However, it is never an equilibrium. The origin of system

$$
\dot{z}=-z^{2}(\varepsilon-z)
$$

is $\operatorname{PSGAS}$ when $\varepsilon \rightarrow 0$. However, it is an unstable equilibrium for every $\varepsilon$.

In previous papers we adopted the following terminolgy : a point which is PSGAS was said to be seemingly GAS (see [16], Section 5.1 or [19], Section 3.2). Since this notion is strongly related to the notion of practical stabilizability introduced by Byrnes and Isidori [3] we prefer to adopt here the terminology PSGAS (see also [17], Section 2.4).

\subsection{Instantaneous Stability and Uniform Infinitesimal Boundedness}

In Section 4.2 we introduced the nonstandard concepts of IS and UIB for system (4). Let us give their standard formulation for the system (1) depending on the parameter $\varepsilon$.

Definition 5.4 The origin of system $\dot{y}=G(y, \varepsilon)$ is $I S$ when $\varepsilon \rightarrow 0$, if for every $\delta>0$, $A>0$ and $t_{0}>0$, there exists $\varepsilon_{0}>0$ such that whenever an initial condition $y_{0}$ satisfies $\left\|y_{0}\right\| \leq A$, it follows that $\left\|y^{G}\left(t, y_{0}, \varepsilon\right)\right\|<\delta$ for all $t \geq t_{0}$ and all $\left.\varepsilon \in\right] 0, \varepsilon_{0}[$.

Definition 5.5 The system (1) is UIB when $\varepsilon \rightarrow 0$, if for every $A>0$, there exist $B>0$, $t_{0}>0$ and $\varepsilon_{0}>0$ such that whenever an initial condition $\left(x_{0}, y_{0}\right)$ satisfies $\left\|x_{0}\right\| \leq A$ and $\left\|y_{0}\right\| \leq A$, it follows that $\left\|x^{(f, G)}\left(t,\left(x_{0}, y_{0}\right), \varepsilon\right)\right\| \leq B$ for all $t \in\left[0, t_{0}\right]$ and all $\varepsilon \in] 0, \varepsilon_{0}[$.

We consider the condition below

H6 : the origin of system $\dot{y}=G(y, \varepsilon)$ is IS and system (1) is UIB when $\varepsilon \rightarrow 0$. 
Theorem 5.6 Assume that HI and H6 hold. Then the origin of system (1) is PSGAS when $\varepsilon \rightarrow 0$.

This result is a corollary of Theorem 4.8 (see Appendix D).

\section{3. $\omega$-Instantaneous Stability and $\omega$-Uniform Infinitesimal Boundedness}

In Section 4.3 we introduced the nonstandard concepts of $\omega$-IS and $\omega$-UIB for system (4), where $\omega$ is some unlimited positive real number. Let us give their standard formulation for the system (1) depending on the parameter $\varepsilon$. Let $\omega(\varepsilon)$ be a positive function satisfying $\lim _{\varepsilon \rightarrow 0} \omega(\varepsilon)=+\infty$.

Definition 5.7 The origin of system $\dot{y}=G(y, \varepsilon)$ is $\omega$-IS when $\varepsilon \rightarrow 0$, if for every $\delta>0$ and $A>0$, there exist $\varepsilon_{0}>0$ and $\tau_{0}>0$ such that whenever an initial condition $y_{0}$ satisfies $\left\|y_{0}\right\| \leq A$, it follows that $\left\|y^{G}\left(\tau / \omega(\varepsilon), y_{0}, \varepsilon\right)\right\|<\delta$ for all $\tau>\tau_{0}$ and $\left.\varepsilon \in\right] 0, \varepsilon_{0}[$.

Definition 5.8 The system (1) is $\omega$-UIB when $\varepsilon \rightarrow 0$, if for every $A>0$ and $s>0$, there exist $B>0$ and $\varepsilon_{0}>0$ such that whenever an initial condition $\left(x_{0}, y_{0}\right)$ satisfies $\left\|x_{0}\right\| \leq$ $A$ and $\left\|y_{0}\right\| \leq A$, it follows that $\left\|x^{(f, G)}\left(t,\left(x_{0}, y_{0}\right), \varepsilon\right)\right\| \leq B$ for all $t \in[0, s / \omega(\varepsilon)]$ and $\varepsilon \in] 0, \varepsilon_{0}[$.

We consider the condition below.

H7 : there exists a positive function $\omega(\varepsilon)$ such that $\lim _{\varepsilon \rightarrow 0} \omega(\varepsilon)=+\infty$, (1) is $\omega$-UIB when $\varepsilon \rightarrow 0$ and the origin of system $\dot{y}=G(y, \varepsilon)$ is $\omega$-IS when $\varepsilon \rightarrow 0$.

Theorem 5.9 Assume that H1 and H7 hold. Then the origin of (1) is PSGAS when $\varepsilon \rightarrow 0$.

This result is a corollary of Theorem 4.13 (see Appendix D).

\section{Applications}

\subsection{Equivalence between $\mathrm{H} 4$ and $\mathrm{H} 5$}

The difference between Theorems 4.8 and 4.13 is that the first result requires assumption $\mathrm{H} 4$, that is (IS)+(UIB), while the second result requires assumption H5, that is $(\omega$ IS $)+(\omega$-UIB $)$. Apparently these two assumptions are not equivalent since we have

$$
\omega-I S \Rightarrow I S \text { and } U I B \Rightarrow \omega-U I B .
$$

Notice that in general $\omega$-UIB does not imply UIB. Indeed consider the system

$$
\dot{x}=y \quad \dot{y}=\omega
$$

where $\omega \simeq+\infty$. Let $x(t)=x_{0}+\omega t^{2} / 2+y_{0} t$ be the solution with limited initial condition $\left(x_{0}, y_{0}\right)$. Then $x(t)$ is limited for all $t \in[0, s / \omega]$ where $s=\sqrt{\omega}$, that is, the system is $\omega$-UIB. However, $x\left(1 / \omega^{1 / 4}\right)$ is not limited, that is, the system is not UIB.

We have $I S \Rightarrow \omega-I S$, for some $\omega \simeq+\infty$, and $H 5 \Rightarrow U I B$ as it is shown in the following Lemmas. 
Lemma 6.1 If the origin of $\dot{y}=g(y)$ is IS then there exists $\omega$ unlimited such that it is $\omega-I S$.

Proof. Since $y\left(t, y_{0}\right) \simeq 0$ for all limited $y_{0}$ and all non infinitesimal positive $t$, the set

$$
E=\left\{l>0:\left\|y\left(t, y_{0}\right)\right\| \leq 1 / l \text { for all } t \geq 1 / l \text { and }\left\|y_{0}\right\| \leq l\right\}
$$

contains all limited $l>0$. By the Cauchy Principle (see Appendix A), it contains some unlimited $\omega$. Hence, for all $\left\|y_{0}\right\| \leq \omega$ (and a fortiori for all limited $x_{0}$ ), for all $t \geq 1 / \omega$ (and $a$ fortiori for all $t=\tau / \omega$ with $\tau$ unlimited), the solution satisfies $\left\|y\left(t, y_{0}\right)\right\| \leq 1 / \omega$ (and a fortiori $y\left(t, y_{0}\right) \simeq 0$ ). That is, the origin of (5) is $\omega$-IS.

Lemma 6.2 If H5 holds then system (4) is UIB.

Proof. Let $\left(x_{0}, y_{0}\right)$ be limited and let $x(t)=x^{(f, g)}\left(t,\left(x_{0}, y_{0}\right)\right)$. By the first part of Theorem 4.12, we have that $x(t)$ is limited for all infinitesimal positive $t$.

From (19) and Lemma 6.1 we deduce that $H 4 \Rightarrow H 5$. From (19) and Lemma 6.2 we deduce that $H 5 \Rightarrow H 4$. Hence, $H 5 \Leftrightarrow H 6$. Let us show how to use both conditions $\mathrm{H} 5$ and H6 in the proof of Theorem 2.2. Recall that this theorem was already proven in Section 2 as a corollary of Tikhonov's theorem.

Proof. [of Theorem 2.2] The result follows from Theorem 4.8. Since $\varepsilon \simeq 0$ and $\mathrm{H} 2$ holds, the origin of the second equation in system (6) is IS. Let us prove that system $(1,6)$ is UIB. Let $(x(t), y(t))$ be the solution of system (6) with initial condition $\left(x_{0}, y_{0}\right)$. By $\mathrm{H} 2, y(s)$ is limited for every $s \geq 0$. By Proposition B.2, $x(t)$ is limited for every positive infinitesimal $t$. Hence, (6) is UIB.

The result also follows from Theorem 4.13. Since $\varepsilon \simeq 0$ and $\mathrm{H} 2$ holds, the origin of the second equation in system (6) is $(1 / \varepsilon)$-IS. Let us prove that (6) is $(1 / \varepsilon)$-UIB. Let $\left(x_{0}, y_{0}\right)$ be limited. Define $\xi(\tau)=x(\varepsilon \tau)$ and $\eta(\tau)=y(\varepsilon \tau)$, where $(x(t), y(t))$ is the solution of system (6). System (6) becomes

$$
\xi^{\prime}=\varepsilon f(\xi, \eta) \quad \eta^{\prime}=G(\eta)
$$

where $^{\prime}=d / d \tau$. By Lemma B.1, we have $\xi(\tau) \simeq x_{0}$ as long as $\tau$ is limited. By Lemma A.2, there exists an unlimited $s$ such that $\xi(\tau) \simeq x_{0}$ for all $0 \leq \tau \leq s$. Thus, $x(t)=\xi(t / \varepsilon)$ is limited for all $t \in[0, \varepsilon s]$. Hence, $(6)$ is $(1 / \varepsilon)$-UIB.

\subsection{Steadiness of control systems}

The difficulty in applying Theorem 4.8 or 4.13 is to decide whether system (4) is UIB (resp. $\omega$-UIB) or not. Let us give some sufficient conditions for UIB. When $f$ is a standard function and the control $u$ is limited, we know, from Proposition B.1, that for every limited initial condition $x_{0}$ and every infinitesimal $t$, the solution $x^{f}\left(t, x_{0}, u(\cdot)\right)$ of the control system (5) is defined and limited. This property does not hold if the control $u$ is not limited. Consider, for example, the control system

$$
\dot{x}=u
$$

and take $u(t)=\gamma$, with $\gamma$ unlimited. Here, we have $x(1 / \sqrt{\gamma}, 0, u(\cdot))=\sqrt{\gamma}$ which is not limited. This is the reason why we introduce the definition below. 
Definition 6.3 Let u be a control. A control system $f$ is u-steady if for every limited $x_{0}$ and every positive infinitesimal $t, x^{f}\left(t, x_{0}, u(\cdot)\right)$ is limited.

Hence, (4) is UIB if and only if the control system $\dot{x}=f(x, y)$ is $y$-steady for every control $y(t)=y^{g}\left(t, y_{0}\right)$ produced by $\dot{y}=g(y)$, where $y_{0}$ is limited.

Example. The control system $\dot{x}=-u x^{3} / 2$ is not $u$-steady for the control $u$ defined by

$$
u(t)=1-\gamma^{2} t \mathrm{e}^{-\gamma t}
$$

where $\gamma$ is unlimited. Indeed, we have

$$
x\left(t, x_{0}, u(\cdot)\right)=\frac{x_{0}}{\sqrt{1+x_{0}^{2}\left[t-1+(\gamma t+1) \mathrm{e}^{-\gamma t}\right]}}
$$

and $x\left(t, x_{0}, u(\cdot)\right)$ escapes to infinity in an infinitesimal time $t_{e}$ as long as $x_{0}>1$.

The control system $\dot{x}=-u x$ is $u$-steady for the control $u$ defined by (20). Indeed, we have

$$
x\left(t, x_{0}, u(\cdot)\right)=x_{0} \exp \left(1-t-(\varepsilon t+1) \mathrm{e}^{-\varepsilon t}\right)
$$

and $x\left(t, x_{0}, u(\cdot)\right)$ is limited for all limited $x_{0}$ and all $t \simeq 0$.

Proposition 6.4 1) If $\|f\|$ is bounded on $\mathbb{R}^{n} \times \mathbb{R}^{m}$ by a limited value $M$ then $f$ is $u$ steady for every control $u$.

2) If there exists a limited real number $C$, such that for every $(x, u) \in \mathbb{R}^{n+m}$ we have $<x, f(x, u)>\leq C\left(1+\|x\|^{2}\right)$, then $f$ is u-steady for every control $u$.

Proof. 1) From $x(t)=x_{0}+\int_{0}^{t} f(x(s), u(s)) d s$ we obtain that $\|x(t)\| \leq\left\|x_{0}\right\|+t M$. Thus $x(t)$ is limited for all limited $x_{0}$ and all $t \simeq 0$.

2) Let $\delta(t)=\|x(t)\|^{2} / 2$ then $\delta^{\prime}(t)=<x(t), f(x(t), u(t))>\leq C(1+2 \delta(t))$. Thus $\delta(t) \leq \delta(0) \mathrm{e}^{2 C t}+\left(\mathrm{e}^{2 C t}-1\right) / 2$ and $\delta(t)$ is limited for all limited $x_{0}$ and all $t \simeq 0$.

Definition 6.5 Let $p \in \mathbb{N}$. A control $u$ is infinitesimally of class $\mathcal{L}^{p}$ if $\int_{0}^{t}\|u(s)\|^{p} d s$ is limited for all infinitesimal positive $t$. A control $u$ is of class $\mathcal{L}^{p}$ if $\int_{0}^{t}\|u(s)\|^{p} d s$ is limited for all limited positive $t$.

Proposition 6.6 Assume that $f(x, u)=u X(x)$ where $u \in \mathbb{R}$ and $X$ is a standard complete vector field on $\mathbb{R}^{n}$. Then $f$ is u-steady for every control $u$ which is infinitesimally of class $\mathcal{L}^{1}$.

Proof. We have $x^{f}(t, u(\cdot))=X_{v(t)}\left(x_{0}\right)$ where $X_{t}$ is the flow of the vector field $X$ and $v(t)=\int_{0}^{t} u(s) d s$. Thus $x(t)$ is limited for all limited $x_{0}$ and all positive infinitesimal $t$.

Proposition 6.7 Assume that $f(x, u)=u_{1} X_{1}(x)+u_{2} X_{2}(x)$ where $u=\left(u_{1}, u_{2}\right) \in \mathbb{R}^{2}$ and $X_{1}, X_{2}$ are vector fields on $\mathbb{R}^{n}$. Assume there exists a limited real number $L$ such that for all $x \in \mathbb{R}^{n}$ we have $\left\langle x, X_{1}(x)>\leq L\right.$ and $<x, X_{2}(x)>\leq L$. Then $f$ is $u$-steady for every control $u$ which is infinitesimally of class $\mathcal{L}^{1}$. 
Proof. Let $\delta(t)=\|x(t)\|^{2} / 2$ then we have

$$
\delta^{\prime}(t)=<x(t), f(x(t), u(t))>\leq L\left(\left|u_{1}(t)\right|+\left|u_{2}(t)\right|\right) .
$$

Thus $\delta(t) \leq \delta(0)+L \int_{0}^{t}\|u(s)\| d s$ is limited for all limited $x_{0}$ and all positive infinitesimal $t$.

Theorem 6.8 Assume that $f$ is standard, and satisfies that $f$ is bounded on $\mathbb{R}^{n} \times \mathbb{R}^{m}$ or there exists a real number $C$ such that for every $(x, u) \in \mathbb{R}^{n+m}$ we have $\langle x, f(x, u)\rangle \leq$ $C\left(1+\|x\|^{2}\right)$. Assume that HI holds and the origin of $\dot{y}=g(y)$ is IS. Then the origin of $(1,4)$ is S-GAS.

Proof. The result follows from Theorem 4.8 By Proposition 6.4, $f$ is $u$-steady for every control $u$. Hence (4) is UIB and since the origin of $\dot{y}=g(y)$ is IS it follows that H4 holds.

Theorem 6.9 Assume that $f$ is standard, and satisfies that $f(x, y)=\varphi(y) X(x)$ where $\varphi: \mathbb{R}^{m} \rightarrow \mathbb{R}$ is standard and $X$ is a standard complete vector field on $\mathbb{R}^{n}$. Assume that $H 1$ holds, the origin of $\dot{y}=g(y)$ is IS and for every limited $y_{0}$ the control $\varphi\left(y^{g}\left(\cdot, y_{0}\right)\right)$ is infinitesimally of class $\mathcal{L}^{1}$. Then the origin of (4) is s-GAS.

Proof. The result follows from Theorem 4.8. By Proposition 6.6, $f$ is $u$-steady for every control $u(t)=\varphi\left(y^{g}\left(t, y_{0}\right)\right)$ where $y_{0}$ is limited. Hence (4) is UIB and since the origin of $\dot{y}=g(y)$ is IS it follows that H4 holds.

Theorem 6.10 Assume that $f(x, y)=\varphi_{1}(y) X_{1}(x)+\varphi_{2}(y) X_{2}(x)$ where $\varphi=\left(\varphi_{1}, \varphi_{2}\right)$ : $\mathbb{R}^{m} \rightarrow \mathbb{R}^{2}$ is a standard function, and $X_{1}, X_{2}$ are standard vector fields on $\mathbb{R}^{n}$. Assume there exists a real number $L$ such that for all $x \in \mathbb{R}^{n}$ we have $\left\langle x, X_{1}(x)\right\rangle \leq L$ and $<x, X_{2}(x)>\leq L$. Assume that HI holds, the origin of $\dot{y}=g(y)$ is IS and for every limited $y_{0}$ the control $\varphi\left(y^{g}\left(\cdot, y_{0}\right)\right)$ is infinitesimally of class $\mathcal{L}^{1}$. Then the origin of system (4) is S-GAS.

Proof. The result follows from Theorem 4.8. By Proposition 6.7, $f$ is $u$-steady for every control $u(t)=\varphi\left(y^{g}\left(t, y_{0}\right)\right)$ where $y_{0}$ is limited. Hence (4) is UIB and since the origin of $\dot{y}=g(y)$ is IS it follows that H4 holds.

Let us end this section by stating a few properties of controls and some criteria that insure that a control is infinitesimally of class $\mathcal{L}^{1}$.

Lemma 6.11 If an impulse control $u$ is infinitesimally of class $\mathcal{L}^{p}$ then it is of class $\mathcal{L}^{p}$.

Proof. Let $u$ be an impulse control. Then $u(t) \simeq 0$ for all $t \gg 0$. By Lemma A.2, there exists $t_{1} \simeq 0$ such that $u(t) \simeq 0$ for all $t \geq t_{1}$. Let $t$ be positive limited. Then we have

$$
\int_{0}^{t}\|u(s)\|^{p} d s=\int_{0}^{t_{1}}\|u(s)\|^{p} d s+\int_{t_{1}}^{t}\|u(s)\|^{p} d s .
$$

Since $u$ is infinitesimally of class $\mathcal{L}^{p}$, the first integral in the right hand side is limited. Since $t-t_{1}$ is limited and $u(s) \simeq 0$ on $\left[t_{1}, t\right]$, the second integral in the right hand side is infinitesimal. Thus $\int_{0}^{t}\|u(s)\|^{p} d s$ is limited for all limited positive $t$. 
Definition 6.12 Let $\alpha$ be a positive constant. A function $t \rightarrow u(t)$ is said to be $\alpha$-bounded if $u(t) / \alpha$ is limited for all $t \geq 0$.

Lemma 6.13 Let $u$ be a control. Assume that $u$ is an $\omega$-impulse and that it is $\omega$-bounded. Then, $u$ is of class $\mathcal{L}^{1}$.

Proof. Let $a>0$ be standard. Since $u(\tau / \omega) \simeq 0$ for every $\tau \simeq+\infty$, the set $\{s: \forall \tau \geq$ $s\|u(\tau / \omega)\| \leq a\}$ contains every $s \simeq+\infty$. By the Cauchy Principle (Lemma A.1), it contains some limited $s_{0}$. Let $t$ be limited. Then we have

$$
\int_{0}^{t}\|u(s)\| d s=\int_{0}^{s_{0} / \omega}\|u(s)\| d s+\int_{s_{0} / \omega}^{t}\|u(s)\| d s .
$$

Since $u$ is $\omega$-bounded, there exists $A$ limited such that $\|u(s)\| \leq \omega A$ for all $s \in\left[0, s_{0} / \omega\right]$ and hence the first integral in the right hand side is limited. Since $t-s_{0} / \omega$ is limited, the second integral in the right hand side is also limited. Thus, $\int_{0}^{t}\|u(s)\| d s$ is limited.

\section{7. $\alpha$-Boundedness and Peaking}

Definition 7.1 Let $\alpha$ be a positive constant. The system $\dot{y}=g(y)$ is $\alpha$-bounded if for every limited initial condition $y_{0}, \frac{1}{\alpha} y^{g}\left(t, y_{0}\right)$ is limited for all $t \geq 0$.

Proposition 7.2 If the system $\dot{y}=g(y)$ is $\alpha$-bounded and its origin is S-GAS then, for all $\gamma \simeq+\infty$, the system $\dot{y}=\gamma g(y)$ is $\alpha$-bounded and its origin is $\gamma$-IS. In particular, if $G$ is standard and H2 holds then system $\dot{y}=\gamma G(y)$ is 1-bounded and its origin is $\gamma$-IS.

Proof. Multiplying the vector field by $\varepsilon$ does not change the trajectories, but only the velocity. Moreover, if $G$ is standard and $\mathrm{H} 2$ holds then, according to Proposition 4.3, the system $\dot{y}=G(y)$ is 1 -bounded and its origin is S-GAS.

Definition 7.3 The system $\dot{y}=g(y)$ exhibits a peaking of order $\alpha$ if it is $\alpha$-bounded and there exists a limited $y_{0}$ and a positive $t$ such that $y^{g}\left(t, y_{0}\right) / \alpha$ is non infinitesimal.

Example. The second equation in system (11) exhibits a peaking of order $1 / \varepsilon$.

Proposition 7.4 Consider the system

$$
\dot{y}_{1}=y_{2}, \dot{y}_{2}=y_{3}, \cdots, \dot{y}_{n-1}=y_{n}, \dot{y}_{n}=h(y, \varepsilon) \text {, }
$$

where $h(0, \varepsilon)=0$. Assume that the origin is $\omega-I S$. Then the peaking is at least of order $\omega^{n-1}$.

Proof. We do the proof for $n=2$. The general case is similar. Assume that $y_{1}(0)=1$ and for every positive unlimited $\tau$ we have $y_{1}(\tau / \omega) \simeq 0$. Let $M=\sup _{t \geq 0}\left|y_{2}(t)\right|$. Since $y_{1}(t)=1+\int_{0}^{t} y_{2}(s) d s$, we have, for every positive unlimited $\tau$,

$$
\frac{1}{2} \leq \int_{0}^{\tau / \omega}\left|y_{2}(s)\right| d s \leq M \tau / \omega
$$


Thus $M \geq \omega /(2 \tau)$ for all positive unlimited $\tau$. By the Cauchy Principle (see Appendix A), there exists an appreciabe $a$ such that $M \geq a \omega$, that is, the peaking is at least of order $\omega$.

Let us now examine the peaking phenomenon in a nonlinear system.

Example. Consider the singularly perturbed forced Van der Pol equation

$$
\varepsilon \ddot{y}+\dot{y}\left(y^{2}-1\right)+y=a
$$

where $a>1$. It is well known that $(y=a, \dot{y}=0)$ is a GAS equilibrium. In the Lienard space $\left(y_{1}=y-a, v=\varepsilon \dot{y}+y^{3} / 3-y-a^{3} / 3+a\right)$, equation (21) becomes

$$
\varepsilon \dot{y}_{1}=v-\left(y_{1}+a\right)^{3} / 3+\left(y_{1}+a\right)+a^{3} / 3-a, \quad \dot{v}=-y_{1} .
$$

According to Tikhonov's theorem, system (22) is 1-bounded and its origin is S-GAS. In the phase space $\left(y_{1}=y-a, y_{2}=\dot{y}\right)$, equation (21) becomes

$$
\dot{y}_{1}=y_{2}, \quad \varepsilon \dot{y}_{2}=-y_{1}-y_{2}\left(\left(y_{1}+a\right)^{2}-1\right) \text {. }
$$

From the above analysis in the Lienard plane, we see that system (23) is $\gamma$-bounded and its origin is S-GAS, where $\gamma=\varepsilon^{-1}$. Consider now the system

$$
\dot{y}_{1}=\varepsilon y_{2}, \quad \dot{y}_{2}=-\varepsilon^{2}\left(y_{1}+y_{2}\left(\left(y_{1}+a\right)^{2}-1\right)\right),
$$

obtained by multiplying by $\varepsilon$ the vector field associated to system (23). Applying Proposition 7.2, we see that system (24) is $\varepsilon$-bounded and its origin is $\varepsilon$-IS. This system exhibits a peaking of order $\varepsilon$.

Let us now investigate the properties of a linear system

$$
\dot{y}=G(\varepsilon) y,
$$

under condition H3. If $G(\varepsilon)=\frac{1}{\varepsilon} G$, where $G$ is a standard Hurwitz matrix, then, by Proposition 7.2, system (25) is 1-bounded and its origin is $(1 / \varepsilon)$-IS. However, it must be noted that condition $\mathrm{H} 3$ does not imply that the origin of system (25) is IS, as it is shown by the following examples.

Example. Consider system (25) where

$$
G(\varepsilon)=\left(\begin{array}{cc}
\alpha-\varepsilon & 1 \\
-\varepsilon^{2} & -\alpha-\varepsilon
\end{array}\right), \quad \alpha \in \mathbb{R}
$$

Since both eigenvalues of $G(\varepsilon)$ are equal to $-1 / \varepsilon$, condition $\mathrm{H} 3$ is satisfied. The exponential matrix

$$
\mathrm{e}^{t G(\varepsilon)}=\left(\begin{array}{cc}
1+\alpha t & t \\
-\alpha^{2} t & 1-\alpha t
\end{array}\right) \mathrm{e}^{-t / \varepsilon}
$$

shows that as $\varepsilon \rightarrow 0$, the solution $y(t, \varepsilon)=\mathrm{e}^{t G} y_{0}$ will decay to zero arbitrarily fast. However, if $\alpha$ is sufficiently large, the origin is not IS, even if $\varepsilon$ is infinitesimal positive.

Example. Consider system (25) where

$$
G(\varepsilon)=\left(\begin{array}{cc}
0 & 1 \\
-1 / \varepsilon^{2}-\sigma^{2} & -2 / \varepsilon
\end{array}\right), \quad \sigma \in \mathbb{R}
$$


Since the eigenvalues of $G(\varepsilon)$ are equal to $-(1 / \varepsilon) \pm \mathrm{i} \sigma$, condition $\mathrm{H} 3$ is satisfied. The exponential matrix

$$
\mathrm{e}^{t G(\varepsilon)}=\left(\begin{array}{cc}
\cos (\sigma t)+\frac{\sin (\sigma t)}{\varepsilon \sigma} & \frac{\sin (\sigma t)}{\sigma} \\
-\left(\sigma+\varepsilon^{2} \sigma^{2}\right) \frac{\sin (\sigma t)}{\varepsilon^{2} \sigma} & \cos (\sigma t)-\frac{\sin (\sigma t)}{\varepsilon \sigma}
\end{array}\right) \mathrm{e}^{-t / \varepsilon}
$$

shows that as $\varepsilon \rightarrow 0$, the solution $y(t, \varepsilon)=\mathrm{e}^{t G} y_{0}$ will decay to zero arbitrarily fast. However, if $\sigma$ is sufficiently large, the origin is not IS, even if $\varepsilon$ is infinitesimal positive.

This last system is in the Luenberger-Brunowsky (LB) canonical form. It turns out that if we make a supplementary assumption on the spectrum of a system in the LB canonical form, then its origin is IS and we can evaluate the peaking of the system. Consider a system in the LB canonical form:

$$
\dot{y}_{1}=y_{2}, \dot{y}_{2}=y_{3}, \cdots, \dot{y}_{n-1}=y_{n}, \dot{y}_{n}=a_{1} y_{1}+a_{2} y_{2}+\cdots+a_{n} y_{n} .
$$

Theorem 7.5 We assume that the spectrum of system (26) is of order $-\gamma$, that is, every eigenvalue $\lambda_{k}$ is of the form $\lambda_{k}=\gamma c_{k}$ where $c_{k}$ is a limited complex number with appreciable negative real part. Then the system is $\gamma^{n-1}$-bounded and if $\gamma$ is unlimited positive, its origin is IS.

Proof. The eigenvalues are the roots of the polynomial

$$
P(z)=\prod_{i=1}^{n}\left(z-\lambda_{i}\right)=z^{n}-a_{n} z^{n-1}-a_{n-1} z^{n-2}-a_{2} z-a_{1} .
$$

The numbers $a_{i}$ are given in terms of the $\lambda_{i}$ by means of the formula

$$
a_{k}=(-1)^{n-k} \sum_{j_{1}<\cdots<j_{n+1-k}} \lambda_{j_{1}} \cdots \lambda_{j_{n+1-k}} .
$$

Since $\lambda_{k}=c_{k} \gamma$ for all $k$, we have $a_{k}=\gamma^{n+1-k} b_{k}$ where

$$
b_{k}=(-1)^{n-k} \sum_{j_{1}<\cdots<j_{n+1-k}} c_{j_{1}} \cdots c_{j_{n+1-k}}
$$

are limited. Let $\eta_{k}(\tau)=\gamma^{1-k} y_{k}(\tau / \gamma)$, for $k=1 \cdots n$, so that (26) becomes

$$
\eta_{1}^{\prime}=\eta_{2}, \eta_{2}^{\prime}=\eta_{3}, \cdots, \eta_{n-1}^{\prime}=\eta_{n}, \eta_{n}^{\prime}=b_{1} \eta_{1}+b_{2} \eta_{2}+\cdots+b_{n} \eta_{n}
$$

where $^{\prime}=d / d \tau$. The eigenvalues of (27) are $\lambda_{k} / \gamma=c_{k}$. Therefore there exist two appreciable positive numbers $K$ and $\alpha$ such that

$$
\|\eta(\tau)\| \leq K \mathrm{e}^{-\alpha \tau}\|\eta(0)\| \quad \text { for } \tau \geq 0
$$

where $\|\eta\|=\sum_{k=1}^{n}\left|\eta_{k}\right|$. Since $\|\eta(\tau)\| \leq\|y(\tau / \gamma)\| \leq \gamma^{n-1}\|\eta(\tau)\|$ we have

$$
\|y(t)\| \leq K \gamma^{n-1} \mathrm{e}^{-\gamma \alpha t}\|y(0)\| \quad \text { for } t \geq 0 .
$$

Hence (26) is $\gamma^{n-1}$-bounded and if $\gamma$ is unlimited positive, its origin is IS. 


\section{Further developments}

Thus far, we considered only triangular systems, in order to focus on the new concepts of IS and UIB without being burdened by technicalities. The second equation does not contain the first variable and the behavior of $y$ does not depend on $x$. There are many applications for this particular case. There are also more realistic problems where the second equation in system (4) depends on $x$ also

$$
\dot{x}=f(x, y), \quad \dot{y}=g(x, y),
$$

and the stability of system (28) is investigated under condition $\mathrm{H} 1$ and the assumption that the origin of the second equation in (28) is IS in some sense to be precised.

For instance, consider the "high gain" observer problem which is well known in automatic control. Let us begin with the linear case which is well understood. We consider the system

$$
\dot{x}=A x+B u, \quad y=C x,
$$

with the usual assumptions of controllability of the pair $(A, B)$ and observability of the pair $(A, C)$. System (29) can be stabilized by $u=R x$ where $R$ is designed such that the matrix $A+B R$ is Hurwitz. Consider now the system

$$
\dot{\hat{x}}=A \hat{x}+B u+K C(\hat{x}-x),
$$

which is called a Luenberger observer [22]. The error between the state $x(t)$ and its observation $\hat{x}(t)$ is $y=\hat{x}-x$ and $y$ is solution of the differential equation

$$
\dot{y}=(A+K C) y .
$$

If $K$ is taken such that the matrix $G:=A+K C$ is Hurwitz, then the error tends to 0 . Moreover, if we assign the eigenvalues of $G$ far to the left in the complex plane, then the origin of system (30) is IS.

Consider now the case where the feedback is based on the estimation given by the Luenberger observer. The full system is

$$
\dot{x}=A x+B R \hat{x}, \quad \dot{\hat{x}}=A \hat{x}+B R \hat{x}+K C(\hat{x}-x),
$$

which can be rewritten using the variables $x$ and $y$ as

$$
\dot{x}=A x+B R(x+y), \quad \dot{y}=(A+K C) y .
$$

This system turns out to be GAS. This can be seen by elementary algebraic considerations, but also through the previous theory, in the case where we chose $K$ such that the origin of the second equation in (31) is IS.

For the nonlinear case, there are circumstances where one can build Luenberger like observers. Let us consider the problem of stabilization of the control system

$$
\dot{x}=\phi(x, u)
$$

under some feedback law $u=R(x)$. Assume that we have designed some $R$ such that system

$$
\dot{x}=\phi(x, R(x))
$$


has the origin as a GAS equilibrium. Assume now that the state vector $x$ is not accessible to measurement, which means that only a certain function $\xi=\varphi(x)$ of the state is available. Is it possible to recover the state $x(t)$ from $\xi(t)$ ? The answer is yes, to some extent. Namely, under some assumptions that we do not detail here [11], there is a system of the form

$$
\dot{\hat{x}}=\vartheta(\hat{x}, \varphi(x))
$$

such that the error $y=\hat{x}-x$ tends to zero as $t \rightarrow+\infty$. Unlike in the linear case (31), the differential equation of the error does contain the variable $x$. Actually one has

$$
\dot{x}=\phi(x, R(\hat{x})), \quad \dot{\hat{x}}=\vartheta(\hat{x}, \varphi(x)),
$$

which can be rewritten in the variables $x$ and $y$ as

$$
\begin{aligned}
& \dot{x}=\phi(x, R(x+y))=f(x, y) \\
& \dot{y}=\vartheta(x+y, \varphi(x))-\phi(x, R(x+y))=g(x, y)
\end{aligned}
$$

Condition H1 is satisfied. If the origin of (33) is IS in some sense, we may hope that the origin of $(32,33)$ is S-GAS. This case is not covered by the results of this paper and thus deserves further attention.

\section{A. Nonstandard Analysis}

In IST we adjoin to ordinary mathematics, say Zermelo Fraenkel Theory with Axiom of Choice (ZFC), a new undefined unary predicate standard (st). The axioms of IST are the usual axioms of ZFC plus three others which govern the use of the new predicate. Hence, all theorems of ZFC remain valid. What is new in IST is an addition, not a change. We call a formula of IST external in the case where it involves the new predicate "st"; otherwise, we call it internal. Thus, internal formulas are the formulas of ZFC. The theory IST is a conservative extension of ZFC, that is, every internal theorem of IST is a theorem of ZFC. Some of the theorems which are proved in IST are external and can be reformulated so that they become internal. Indeed, there is a reduction algorithm which reduces any external formula $A$ of IST to an internal formula $A^{\prime}$, with the same free variables, which satisfies $A \Leftrightarrow A^{\prime}$ for all standard values of the parameters (i.e. the free variables). Here, is the reduction of some frequently occuring formulas [25]:

$$
\begin{aligned}
\forall x\left(\forall^{\text {st }} y P \Rightarrow \forall^{\text {st }} z Q\right) & \Leftrightarrow \forall z \exists^{\text {fin }} y^{\prime} \forall x\left(\forall y \in y^{\prime} P \Rightarrow Q\right) \\
\forall x\left(\exists^{\text {st }} w \forall^{\text {st }} y P \Rightarrow \forall^{\text {st }} z Q\right) & \Leftrightarrow \forall w \forall \exists^{\mathrm{fin}} y^{\prime} \forall x\left(\forall y \in y^{\prime} P \Rightarrow Q\right) \\
\forall x\left(\exists^{\text {st }} w \forall^{\text {st }} y P \Rightarrow \exists^{\text {st }} z Q\right) & \Leftrightarrow \forall w \exists^{\text {fin }} z^{\prime} \exists^{\text {fin }} y^{\prime} \forall x\left(\forall y \in y^{\prime} P \Rightarrow \exists z \in z^{\prime} Q\right)
\end{aligned}
$$

where $P$ and $Q$ are internal formulas whose all parameters are standard. We shall use also

$$
\forall x\left(\exists^{\text {st }} w \forall^{\text {st }} y P \Rightarrow \forall^{\text {st }} t \exists^{\text {st }} z Q\right) \Leftrightarrow \forall w \forall t \exists^{\text {fin }} z^{\prime} \exists^{\text {fin }} y^{\prime} \forall x\left(\forall y \in y^{\prime} P \Rightarrow \exists z \in z^{\prime} Q\right)
$$

A real number $x$ is called infinitesimal when $|x|<a$ for all standard $a>0$, limited when $|x| \leq a$ for some standard $a$, appreciable when it is limited and not infinitesimal, and unlimited, when it is not limited. We use the following notations : $x \simeq 0$ for $x$ infinitesimal, $x \simeq+\infty$ for $x$ unlimited positive, $x \gg 0$ for $x$ non infinitesimal positive. Thus we have

$$
\begin{aligned}
x \simeq 0 & \Leftrightarrow \quad \forall^{\text {st }} a>0|x|<a \\
x \gg 0 & \Leftrightarrow \quad \exists^{\text {st }} a>0 x \geq a \\
x \text { limited } & \Leftrightarrow \quad \exists^{\text {st }} a|x| \leq a \\
x \simeq+\infty & \Leftrightarrow \forall^{\text {st }} a x>a
\end{aligned}
$$


In formulas (34), (35) and (36) we used the following abbreviations

$$
\begin{array}{cl}
\forall^{\mathrm{st}} x A \text { for } \forall x(\operatorname{st}(\mathrm{x}) \Rightarrow \mathrm{A}), & \exists^{\mathrm{st}} \mathrm{xA} \text { for } \exists \mathrm{x}(\operatorname{st}(\mathrm{x}) \& \mathrm{~A}), \\
\forall^{\mathrm{fin}} x A \text { for } \forall x(x \text { finite } \Rightarrow \mathrm{A}), & \exists^{\mathrm{fin}} \mathrm{xA} \text { for } \exists \mathrm{x}(\mathrm{x} \text { finite\&A }) .
\end{array}
$$

Let $(E, d)$ be a standard metric space. Two points $x$ and $y$ in $E$ are called infinitely close, denoted by $x \simeq y$, when $d(x, y) \simeq 0$. A vector in $\mathbb{R}^{n}$ ( $n$ standard) is said to be infinitesimal (respectively limited) if its norm $\|x\|$ is infinitesimal (resp. limited).

We may not use external formulas to define subsets. The notations

$$
\{x \in \mathbb{R}: x \text { is limited }\} \text { or }\{x \in \mathbb{R}: x \simeq 0\}
$$

are not allowed. Moreover, we can prove that

Lemma A.1 (Cauchy principle) There do not exist subsets $L$ and I of $\mathbb{R}$ such that, for all $x$ in $\mathbb{R}, x$ is in $L$ if and only if $x$ is limited, or $x$ is in I if and only if $x$ is infinitesimal.

This result is frequently used in proofs. Suppose that we have shown that a certain internal property $A$ holds for every limited $r$; then we know that $A$ holds for some unlimited $r$, for otherwise we could let $L=\{x \in \mathbb{R}: A\}$. It has the following consequence

Lemma A.2 (Robinson's Lemma). Let $r(t)$ be a real function such that $r(t) \simeq 0$ for all limited $t \geq 0$, then there exists an unlimited positive number $\nu$ such that $r(t) \simeq 0$ for all $t \in[0, \nu]$

Proof. The set of all $s$ such that for all $t \in[0, s]$ we have $|r(t)|<1 / s$ contains all limited $s \geq 1$. By the Cauchy principle, it must contain some unlimited $\nu$.

Lemma A.3 Assume that $F$ is standard. The origin of (17) is stable if and only if $z^{F}\left(t, z_{0}\right) \simeq 0$ for all $t \geq 0$ and all $z_{0} \simeq 0$.

Proof. The characterization of stability given in the lemma is :

$$
\forall z_{0}\left(z_{0} \simeq 0 \Rightarrow \forall t \geq 0 z^{F}\left(t, z_{0}\right) \simeq 0\right) .
$$

Using (36) this formula becomes

$$
\forall z_{0}\left(\forall^{\text {st }} \delta>0\left\|z_{0}\right\|<\delta \Rightarrow \forall^{\text {st }} \varepsilon>0 \forall t \geq 0\left\|z^{F}\left(t, z_{0}\right)\right\|<\varepsilon\right) .
$$

In this formula, $F$ is a standard parameter. By (34), this is equivalent to

$$
\left.\forall \varepsilon>0 \exists^{\text {fin }} \delta^{\prime} \subset\right] 0,+\infty\left[\forall z_{0}\left(\forall \delta \in \delta^{\prime}\left\|z_{0}\right\|<\delta \Rightarrow \forall t \geq 0\left\|z^{F}\left(t, z_{0}\right)\right\|<\varepsilon\right) .\right.
$$

For a finite set $\left.\delta^{\prime} \subset\right] 0,+\infty\left[, \forall \delta \in \delta^{\prime}\left\|z_{0}\right\|<\delta\right.$ is the same as $\left\|z_{0}\right\|<\delta$ for $\delta=\min \delta^{\prime}>$ 0 , and so our formula is equivalent to

$$
\forall \varepsilon>0 \exists \delta>0 \forall z_{0}\left(\left\|z_{0}\right\|<\delta \Rightarrow \forall t \geq 0\left\|z^{F}\left(t, z_{0}\right)\right\|<\varepsilon\right) .
$$

This is the usual definition of stability. 
Lemma A.4 Assume that $F$ is standard. The origin of (17) is globally attractive if and only if $z^{F}\left(t, z_{0}\right) \simeq 0$ for all positive unlimited $t$ and all standard $z_{0} \in \mathbb{R}^{p}$.

Proof. The characterization of global attractivity given in lemma is that for any standard $z_{0} \in \mathbb{R}^{p}$ we have :

$$
\forall t\left(t \simeq+\infty \Rightarrow z^{F}\left(t, z_{0}\right) \simeq 0\right)
$$

Using (36) this formula becomes

$$
\forall t\left(\forall^{\mathrm{st}} T t>T \Rightarrow \forall^{\mathrm{st}} \varepsilon>0\left\|z^{F}\left(t, z_{0}\right)\right\|<\varepsilon\right) .
$$

In this formula, $F$ and $z_{0}$ are standard parameters. By (34), this is equivalent to

$$
\forall \varepsilon>0 \exists^{\mathrm{fin}} T^{\prime} \forall t\left(\forall T \in T^{\prime} t>T \Rightarrow\left\|z^{F}\left(t, z_{0}\right)\right\|<\varepsilon\right) .
$$

For $T^{\prime}$ a finite set, $\forall T \in T^{\prime} t>T$ is the same as $t>T$ for $T=\max T^{\prime}$, and so our formula is equivalent to

$$
\forall \varepsilon>0 \exists T \forall t\left(t>T \Rightarrow\left\|z^{F}\left(t, z_{0}\right)\right\|<\varepsilon\right) .
$$

We have shown that for all standard $z_{0} \in \mathbb{R}^{p}$ (and consequently, by transfer, for all $z_{0} \in$ $\mathbb{R}^{p}$ ) we have $\lim _{t \rightarrow+\infty} z^{F}\left(t, z_{0}\right)=0$. This is the usual definition of global attractivity.

Proof. [of Proposition 4.3] Assume that $F$ is standard and that the origin of (17) is SGAS. Let $z_{0} \simeq 0$. By the continuity of solutions with respect to initial conditions, we have $z\left(t, z_{0}\right) \simeq 0$ for all limited $t \geq 0$. By s-GAS, we have $z\left(t, z_{0}\right) \simeq 0$ for all unlimited $t \geq 0$. Thus, $z\left(t, z_{0}\right) \simeq 0$ for all $t \geq 0$, that is (see Lemma A.3), the origin of (17) is stable. Let $z_{0}$ be standard then $z_{0}$ is limited and by S-GAS, we have $z\left(t, z_{0}\right) \simeq 0$ for all positive unlimited $t$. Thus (see Lemma A.4), the origin of (17) is globally attractive. Hence, it is GAS. Conversely, assume that the origin of (17) is GAS. Let $z_{0}$ be limited. Let $z_{00}$ be standard in $\mathbb{R}^{p}$ such that $z_{0} \simeq z_{00}$. By the continuity of solutions with respect to initial conditions, we have $z\left(t, z_{0}\right) \simeq z\left(t, z_{00}\right)$ for all limited $t \geq 0$. By Lemma A.2, there exists an unlimited $t_{1}$ such that $z\left(t, z_{0}\right) \simeq z\left(t, z_{00}\right)$ for all $0 \leq t \leq t_{1}$. By attractivity (see Lemma A.4), we have $z\left(t, z_{00}\right) \simeq 0$ for all positive unlimited $t$. Thus $z\left(t, z_{0}\right) \simeq 0$ for all unlimited $t \leq t_{1}$. By stability (see Lemma A.3), we have $z\left(t, z_{0}\right) \simeq z\left(t_{1}, z_{0}\right) \simeq 0$ for all $t \geq t_{1}$. Hence, $z\left(t, z_{0}\right) \simeq 0$ for all positive unlimited $t$, that is, the origin of (17) is S-GAS.

Proof. [of Proposition 5.1] By the transfer principle [25], we can assume that $F$ is standard. By Proposition 4.3, we have to prove that the origin of (17) is S-GAS if and only if for all $A>0$, the origin is uniformly attractive with respect to $z_{0}$ for $\left\|z_{0}\right\| \leq A$. This can be seen using the reduction algorithm of external formulas. The definition of S-GAS is :

$$
\forall z_{0} \forall t\left(z_{0} \text { limited } \& t \simeq+\infty \Rightarrow z^{F}\left(t, z_{0}\right) \simeq 0\right) .
$$

Using (36) this formula becomes

$$
\forall z_{0} \forall t\left(\exists^{\text {st }} A\left\|z_{0}\right\| \leq A \& \forall^{\text {st }} T t>T \Rightarrow \forall^{\text {st }} \varepsilon>0\left\|z^{F}\left(t, z_{0}\right)\right\|<\varepsilon\right) .
$$

In this formula, $F$ is a standard parameter. By (34), this is equivalent to

$$
\forall A \forall \varepsilon>0 \exists^{\mathrm{fin}} T^{\prime} \forall z_{0} \forall t\left(\left\|z_{0}\right\| \leq A \& \forall T \in T^{\prime} t>T \Rightarrow\left\|z^{F}\left(t, z_{0}\right)\right\|<\varepsilon\right) .
$$


For $T^{\prime}$ a finite set, $\forall T \in T^{\prime} t>T$ is the same as $t>T$ for $T=\max T^{\prime}$ and so our formula is equivalent to

$$
\forall A \forall \varepsilon>0 \exists T \forall z_{0} \forall t\left(\left\|z_{0}\right\| \leq A \& t>T \Rightarrow\left\|z^{F}\left(t, z_{0}\right)\right\|<\varepsilon\right) .
$$

This is the usual definition of uniform attractivity, with respect to the initial condition $z_{0}$ for $\left\|z_{0}\right\| \leq A$.

\section{B. Nonstandard asymptotic theory}

The classical continuous dependance of solutions with respect to initial conditions and parameters has a beautiful nonstandard translation known in the nonstandard asymptotic theory as the Short Shadow Lemma $[8,21]$. The problem is to compare the solutions of systems

$$
\dot{z}=h_{1}(z, t) \quad \text { and } \quad \dot{z}=h_{0}(z, t)
$$

under the assumptions that the vector field $h_{0}: \mathbb{R}^{p} \times \mathbb{R} \rightarrow \mathbb{R}^{p}$ is standard continuous and has the unicity of the solution with prescribed initial condition, $h_{1}: \mathbb{R}^{p} \times \mathbb{R} \rightarrow \mathbb{R}^{p}$ is continuous and is a regular perturbation of $h_{0}$, that is to say, $h_{1}(z, t) \simeq h_{0}(z, t)$ for all limited $z$ and $t$.

Lemma B.1 (Short Shadow Lemma) Let $t_{0}, t_{1}, z_{0}$ and $z_{1}$ be limited such that $t_{1} \simeq t_{0}$ and $z_{1} \simeq z_{0}$. Let $z_{0}(t)=z^{h_{0}}\left(t, t_{0}, z_{0}\right)$ be the solution of system $\dot{z}=h_{0}(z, t)$ with initial condition $z\left(t_{0}\right)=z_{0}$. Then any solution $z(t)$ of system $\dot{z}=h_{1}(z, t)$ with initial condition $z\left(t_{1}\right)=z_{1}$ is defined as long as $t$ and $z_{0}(t)$ are limited and we have $z(t) \simeq z_{0}(t)$.

Proposition B.2 Let $f(x, u)$ be limited for all limited $x$ and $u$. Let $u$ be a limited control. Let $x_{0}$ be a limited initial condition. Then $x^{f}\left(t, x_{0}, u(\cdot)\right)$ is limited for all infinitesimal positive $t$.

Proof. Let $x(t)=x^{f}\left(t, x_{0}, u(\cdot)\right)$. Assume there exists $t_{1}$ infinitesimal such that $x\left(t_{1}\right)$ is not limited. Let $t_{0}<t_{1}$ be such that $\left\|x\left(t_{0}\right)-x_{0}\right\|=1$ and $\left\|x(t)-x_{0}\right\| \leq 1$ for all $t \in\left[0, t_{0}\right]$. Then $x\left(t_{0}\right)-x_{0}=\int_{0}^{t_{0}} f(x(s), u(s)) d s$ is infinitesimal because we integrate a limited function on an interval of infinitesimal lenght. Thus $x\left(t_{0}\right) \simeq x_{0}$ which contradicts $\left\|x\left(t_{0}\right)-x_{0}\right\|=1$.

Proposition B.3 Let $f$ be standard, $t_{0}$ and $x_{0}$ be limited and $u_{0}$ be a standard control. Assume that $t_{1} \simeq t_{0}, x_{1} \simeq x_{0}$ and $u(t) \simeq u_{0}(t)$ for all $t \geq t_{0}$. For all $t \geq t_{0}$ we have $x^{f}\left(t, t_{0}, x_{0}, u(\cdot)\right) \simeq x^{f}\left(t, t_{0}, x_{0}, u_{0}(\cdot)\right)$ and $x^{f}\left(t, t_{1}, x_{1}, u_{0}(\cdot)\right) \simeq x^{f}\left(t, t_{0}, x_{0}, u_{0}(\cdot)\right)$ as long as $t$ and $x^{f}\left(t, t_{0}, x_{0}, u_{0}(\cdot)\right)$ are limited.

Proof. It is a consequence of Lemma B.1.

Proposition B.4 Let $f$ be standard, $x_{0}$ limited and $u$ a control. Assume there exists $t^{*}$ infinitesimal positive such that $u(t) \simeq 0$ for all $t \geq t^{*}$ and $x^{f}\left(t, x_{0}, u(\cdot)\right)$ limited for $0 \leq t \leq t^{*}$. Let $x_{0}^{*}=x^{f}\left(t^{*}, x_{0}, u(\cdot)\right)$. Then for all $t \geq t^{*}$, we have $x^{f}\left(t, x_{0}, u(\cdot)\right) \simeq$ $x^{f}\left(t, x_{0}^{*}, 0\right)$ as long as $t$ and $x^{f}\left(t, x_{0}^{*}, 0\right)$ are limited. 
Proof. . Using Proposition B.3, we have for all $t \geq t^{*}$ :

$$
\begin{aligned}
x^{f}\left(t, x_{0}, u(\cdot)\right) & =x^{f}\left(t, 0, x_{0}, u(\cdot)\right)=x^{f}\left(t, t^{*}, x_{0}^{*}, u(\cdot)\right) \\
& \simeq x^{f}\left(t, t^{*}, x_{0}^{*}, 0\right) \simeq x^{f}\left(t, 0, x_{0}^{*}, 0\right)=x^{f}\left(t, x_{0}^{*}, 0\right)
\end{aligned}
$$

as long as $t$ and $x^{f}\left(t, x_{0}^{*}, 0\right)$ are limited.

Proposition B.5 Assume that $f$ is standard and that condition HI holds. Let $u$ be a control and let $x(t)=x^{f}\left(t, x_{0}, u(\cdot)\right)$. Assume that there exists $t_{1} \geq 0$ such that $x\left(t_{1}\right) \simeq 0$ and $u(t) \simeq 0$ for $t \geq t_{1}$. Then we have $x(t) \simeq 0$ for all $t \geq t_{1}$.

Proof. Suppose this is not the case, that is, there exists $t>t_{1}$ such that $x(t) \not 0$. Let $t_{2}>t_{1}$ be such that $x_{2}=x\left(t_{2}\right) \not x 0$, and $x(t)$ is limited for all $t_{1} \leq t \leq t_{2}$. If $T=t_{2}-t_{1}$ was limited then, by Proposition B.3,

$$
x_{2}=x^{f}\left(t_{2}, x_{0}, u(\cdot)\right)=x^{f}\left(T, x_{1}, u\left(\cdot+t_{1}\right)\right) \simeq x^{f}\left(T, x_{1}, 0\right) \simeq x^{f}(T, 0,0)=0
$$

which contradicts $x_{2} \neq 0$. Thus, $T$ is infinitely large. By Proposition B.3, we have, for all limited positive $s$

$$
x\left(t_{2}-s\right)=x^{f}\left(t_{2}-s, x_{0}, u(\cdot)\right)=x^{f}\left(-s, x_{2}, u\left(\cdot+t_{2}\right)\right) \simeq x^{f}\left(-s, x_{2}, 0\right) .
$$

By Lemma A.2, this property holds for some unlimited $s$, that can be chosen such that $s \leq T$. Let $t_{3}=t_{2}-s$ then $t_{1} \leq t_{3}$ and $x_{3}=x\left(t_{3}\right)$ is limited. Thus $x_{30}:=x^{f}\left(-s, x_{2}, 0\right)$ is limited also and by Proposition 4.3 we have $x_{2}=x^{f}\left(s, x_{30}, 0\right) \simeq 0$ which contradicts $x_{2} \not 0$.

\section{IS and UIB : equivalence between standard and nonstandard formulations}

Proposition C.1 Assume that $G$ is standard. The origin of system $\dot{y}=G(y, \varepsilon)$ is IS when $\varepsilon \rightarrow 0$ if and only if it is IS for all infinitesimal positive $\varepsilon$.

Proof. To say that the origin of system $\dot{y}=G(y, \varepsilon)$ is IS for all infinitesimal positive $\varepsilon$ is the same as saying that

$$
\forall \varepsilon \forall y_{0} \forall t\left(y_{0} \text { limited \& } t \gg 0 \& \varepsilon \simeq 0 \Rightarrow y^{G}\left(t, y_{0}, \varepsilon\right) \simeq 0\right)
$$

Using (36), this formula becomes

$$
\forall \varepsilon \forall y_{0} \forall t\left(\exists^{\mathrm{st}} A \exists^{\mathrm{st}} t_{0}>0 \forall^{\mathrm{st}} \varepsilon_{0} P \Rightarrow \forall^{\mathrm{st}} \delta>0 Q\right)
$$

where $P$ is the internal formula $\left\|y_{0}\right\| \leq A \& t \geq t_{0} \& \varepsilon<\varepsilon_{0}$ and $Q$ is the internal formula $\left\|y^{G}\left(t, y_{0}, \varepsilon\right)\right\|<\delta$. Here, $G$ is a standard parameter. By (34), this is equivalent to

$$
\forall A \forall \delta>0 \forall t_{0}>0 \exists^{\mathrm{fin}} \varepsilon_{0}^{\prime} \forall \varepsilon \forall y_{0} \forall t_{0}\left(\forall \varepsilon_{0} \in \varepsilon_{0}^{\prime} P \Rightarrow Q\right)
$$


For $\varepsilon_{0}^{\prime}$ a finite set, $\forall \varepsilon_{0} \in \varepsilon_{0}^{\prime} \varepsilon<\varepsilon_{0}$ is the same as $\varepsilon<\varepsilon_{0}$ for $\varepsilon_{0}=\min \varepsilon_{0}^{\prime}$ and so our formula is equivalent to

$$
\forall A \forall \delta>0 \forall t_{0}>0 \exists \varepsilon_{0} \forall \varepsilon \forall y_{0} \forall t\left(\left\|y_{0}\right\| \leq A \& t \geq t_{0} \& \varepsilon>\varepsilon_{0} \Rightarrow\left\|y^{G}\left(t, y_{0}, \varepsilon\right)\right\|<\delta\right) .
$$

This is the usual definition of being IS when $\varepsilon \rightarrow 0$.

Proposition C.2 Assume that $f$ and $G$ are standard. The system (1) is UIB when $\varepsilon \rightarrow 0$ if and only if it is UIB for all infinitesimal positive $\varepsilon$.

Proof. To say system (1) is UIB for all positive infinitesimal $\varepsilon$ is the same as saying that

$$
\begin{aligned}
& \forall \varepsilon \forall\left(x, 0 y_{0}\right) \forall t \geq 0 \\
& \quad\left(x_{0} \text { and } y_{0} \text { limited } \& t \simeq 0 \& \varepsilon \simeq 0 \Rightarrow x^{(f, G)}\left(t,\left(x_{0}, y_{0}\right), \varepsilon\right) \text { limited }\right) .
\end{aligned}
$$

Using (36), this formula becomes

$$
\forall \varepsilon \forall\left(x_{0}, y_{0}\right) \forall t \geq 0\left(\exists^{\mathrm{st}} A \forall^{\mathrm{st}} t_{0}>0 \forall^{\mathrm{st}} \varepsilon_{0} P \Rightarrow \exists^{\mathrm{st}} B Q\right)
$$

where $P$ is the internal formula $\left\|x_{0}\right\| \leq A \&\left\|y_{0}\right\| \leq A \& t \leq t_{0} \& \varepsilon<\varepsilon_{0}$ and $Q$ is the internal formula $\left\|x^{(f, G)}\left(t,\left(x_{0}, y_{0}\right), \varepsilon\right)\right\| \leq B$. Here, $f$ and $G$ are standard parameters. By (34), this is equivalent to

$$
\begin{aligned}
\left.\forall A \exists^{\text {fin }} B^{\prime} \exists^{\text {fin }} t_{0}^{\prime} \subset\right] 0,+\infty\left[\exists^{\text {fin }} \varepsilon_{0}^{\prime} \forall \varepsilon \forall\left(x_{0}, y_{0}\right) \forall t \geq 0\right. & \\
& \left(\forall t_{0} \in t_{0}^{\prime} \forall \varepsilon_{0} \in \varepsilon_{0}^{\prime} P \Rightarrow \exists B \in B^{\prime} Q\right) .
\end{aligned}
$$

For $\varepsilon_{0}^{\prime}$ a finite set, $\forall \varepsilon_{0} \in \varepsilon_{0}^{\prime} \varepsilon<\varepsilon_{0}$ is the same as $\varepsilon<\varepsilon_{0}$ for $\varepsilon_{0}=\min \varepsilon_{0}^{\prime}$. For a finite set $\left.t_{0}^{\prime} \subset\right] 0,+\infty\left[, \forall t_{0} \in t_{0}^{\prime} t \leq t_{0}\right.$ is the same as $t \leq t_{0}$ for $t_{0}=\min t_{0}^{\prime}>0$. For $B^{\prime}$ a finite set, $\exists B \in B^{\prime} Q$ is the same as $Q$ for $B=\max B^{\prime}$ and so our formula is equivalent to

$$
\begin{aligned}
& \forall A \exists B \exists t_{0}>0 \exists \varepsilon_{0} \forall \varepsilon \forall\left(x_{0}, y_{0}\right) \forall t \geq 0 \\
& \quad\left(\left\|x_{0}\right\| \leq A \&\left\|y_{0}\right\| \leq A \& t \leq t_{0} \& \varepsilon<\varepsilon_{0} \Rightarrow\left\|x^{(f, G)}\left(t,\left(x_{0}, y_{0}\right), \varepsilon\right)\right\| \leq B\right) .
\end{aligned}
$$

This is the usual definition of being UIB when $\varepsilon \rightarrow 0$.

Proposition C.3 Assume that $G$ is standard. Let $\omega(\varepsilon)$ be a standard positive function. The origin of system $\dot{y}=G(y \varepsilon)$ is $\omega$-IS when $\varepsilon \rightarrow$ 0if and only if it is $\omega(\varepsilon)$-IS for all infinitesimal positive $\varepsilon$.

Proof. To say that the origin of system $\dot{y}=G(y, \varepsilon)$ is $\omega(\varepsilon)$-IS for all infinitesimal positive $\varepsilon$ is the same as saying that

$$
\forall \varepsilon \forall y_{0} \forall \tau\left(y_{0} \text { limited \& } \tau \simeq+\infty \& \varepsilon \simeq 0 \Rightarrow y^{G}\left(\tau / \omega(\varepsilon), y_{0}, \varepsilon\right) \simeq 0\right) .
$$

Using (36), this formula becomes

$$
\forall \varepsilon \forall y_{0} \forall \tau\left(\exists^{\mathrm{st}} A \forall^{\mathrm{st}} \tau_{0} \forall^{\mathrm{st}} \varepsilon_{0} P \Rightarrow \forall^{\mathrm{st}} \delta>0 Q\right)
$$


where $P$ is the internal formula $\left\|y_{0}\right\| \leq A \& \tau>\tau_{0} \& \varepsilon<\varepsilon_{0}$ and $Q$ is the internal formula $\left\|y^{G}\left(\tau / \omega(\varepsilon), y_{0}, \varepsilon\right)\right\|<\delta$. Here, $G$ and $\omega$ are standard parameters. By (34), this is equivalent to

$$
\forall A \forall \delta>0 \exists^{\mathrm{fin}} \tau_{0}^{\prime} \exists^{\mathrm{fin}} \varepsilon_{0}^{\prime} \forall \varepsilon \forall y_{0} \forall \tau\left(\forall \tau_{0} \in \tau_{0}^{\prime} \forall \varepsilon_{0} \in \varepsilon_{0}^{\prime} P \Rightarrow Q\right) .
$$

For $\varepsilon_{0}^{\prime}$ a finite set, $\forall \varepsilon_{0} \in \varepsilon_{0}^{\prime} \varepsilon<\varepsilon_{0}$ is the same as $\varepsilon<\varepsilon_{0}$ for $\varepsilon_{0}=\min \varepsilon_{0}^{\prime}$. For $\tau_{0}^{\prime}$ a finite set, $\forall \tau_{0} \in \tau_{0}^{\prime} \tau>\tau_{0}$ is the same as $\tau>\tau_{0}$ for $\tau_{0}=\max \tau_{0}^{\prime}$ and so our formula is equivalent to

$$
\begin{aligned}
& \forall A \forall \delta>0 \exists \tau_{0} \exists \varepsilon_{0} \forall \varepsilon \forall y_{0} \forall \tau \\
& \qquad\left(\left\|y_{0}\right\| \leq A \& \tau>\tau_{0} \& \varepsilon>\varepsilon_{0} \Rightarrow\left\|y^{G}\left(\tau / \omega(\varepsilon), y_{0}, \varepsilon\right)\right\|<\delta\right) .
\end{aligned}
$$

This is the usual definition of being $\omega$-IS when $\varepsilon \rightarrow 0$.

Proposition C.4 Assume that $f$ and $G$ are standard. Let $\omega(\varepsilon)$ be a standard positive functions. The system (1) is $\omega$-UIB when $\varepsilon \rightarrow 0$ if and only if it is $\omega(\varepsilon)$-UIB for all positive infinitesimal $\varepsilon$.

Proof. To say that system (1) is $\omega(\varepsilon)$-UIB for all positive infinitesimal $\varepsilon$ is the same as saying that

$$
\begin{aligned}
\forall \varepsilon \forall\left(x_{0}, y_{0}\right)\left(\left(x_{0}, y_{0}\right) \text { limited } \& \varepsilon \simeq 0\right. \\
\left.\Rightarrow \exists s \simeq+\infty \forall t \in[0, s / \omega(\varepsilon)] x^{(f, G)}\left(t,\left(x_{0}, y_{0}\right), \varepsilon\right) \text { limited }\right)
\end{aligned}
$$

Using (36), this formula becomes

$$
\forall \varepsilon \forall\left(x_{0}, y_{0}\right)\left(\exists^{\mathrm{st}} A \forall^{\mathrm{st}} \varepsilon_{0} P \Rightarrow \exists s \forall^{\mathrm{st}} s_{0} s \geq s_{0} \forall t \in[0, s / \omega(\varepsilon)] \exists^{\mathrm{st}} B Q\right)
$$

where $P$ is the internal formula $\left\|x_{0}\right\| \leq A \&\left\|y_{0}\right\| \leq A \& \varepsilon<\varepsilon_{0}$ and $Q$ is the internal formula $\left\|x^{(f, G)}\left(t,\left(x_{0}, y_{0}\right), \varepsilon\right)\right\| \leq B$. Here, $f, G$ and $\omega$ are standard parameters. We have

$$
\forall t \in[0, s / \omega(\varepsilon)] \exists^{\text {st }} B Q \Leftrightarrow \exists^{\text {st }} B \forall t \in[0, s / \omega(\varepsilon)] Q .
$$

Indeed, by the idealization principle [25], the left hand side of (38) is equivalent to the formula $\exists^{\text {st fin }} B^{\prime} \forall t \in[0, s / \omega(\varepsilon)] \exists B \in B^{\prime} Q$, which is itself equivalent to the formula $\exists \exists^{\text {st }} B \forall t \in[0, s / \omega(\varepsilon)] Q$, because for $B^{\prime}$ a standard finite set, $\exists B \in B^{\prime} Q$ is the same as $Q$ for $B=\max B^{\prime}$ which is standard. Also, we have

$$
\exists s \forall^{\mathrm{st}} s_{0} s \geq s_{0} \Leftrightarrow \forall^{\mathrm{st}} s_{0} \exists s s \geq s_{0} .
$$

Indeed, by the idealization principle, the left hand side of (39) is equivalent to the formula $\forall^{\text {st fin }} s_{0}^{\prime} \exists s \forall s \in s_{0}^{\prime} s \geq s_{0}$, which is itself equivalent to the formula $\forall^{\text {st }} s_{0} \exists s s \geq s_{0}$, because for a $s_{0}^{\prime}$ a finite set, $\forall s \in s_{0}^{\prime} s \geq s_{0}$ is the same as $s \geq \max s_{0}^{\prime}$ which is standard. Using (38) and (39), we see that formula (37) is equivalent to

$$
\forall \varepsilon \forall\left(x_{0}, y_{0}\right)\left(\exists^{\mathrm{st}} A \forall^{\mathrm{st}} \varepsilon_{0} P \Rightarrow \forall^{\mathrm{st}} s_{0} \exists^{\mathrm{st}} B \exists s \geq s_{0} \forall t \in[0, s / \omega(\varepsilon)] Q\right)
$$

Since we can always take $s=s_{0}$, this formula is equivalent to

$$
\forall \varepsilon \forall\left(x_{0}, y_{0}\right)\left(\exists^{\mathrm{st}} A \forall^{\mathrm{st}} \varepsilon_{0} P \Rightarrow \forall^{\mathrm{st}} s \exists^{\mathrm{st}} B \forall t \in[0, s / \omega(\varepsilon)] Q\right) .
$$


Using (35), this is equivalent to

$$
\forall A \forall s \exists^{\mathrm{fin}} \varepsilon_{0}^{\prime} \exists^{\mathrm{fin}} B^{\prime} \forall \varepsilon \forall\left(x_{0}, y_{0}\right)\left(\forall \varepsilon_{0} \in \varepsilon_{0}^{\prime} P \Rightarrow \exists B \in B^{\prime} \forall t \in[0, s / \omega(\varepsilon)] Q\right)
$$

For $\varepsilon_{0}^{\prime}$ a finite set, $\forall \varepsilon_{0} \in \varepsilon_{0}^{\prime} \varepsilon<\varepsilon_{0}$ is the same as $\varepsilon<\varepsilon_{0}$ for $\varepsilon_{0}=\min \varepsilon_{0}^{\prime}$, for $B^{\prime}$ a finite set, $\exists B \in B^{\prime} Q$ is the same as $Q$ for $B=\max B^{\prime}$ and so our formula is equivalent to

$$
\begin{aligned}
\forall A \forall s \exists \varepsilon_{0} \exists B \forall \varepsilon \forall\left(x_{0}, y_{0}\right)\left(\left\|x_{0}\right\| \leq\right. & A \&\left\|y_{0}\right\| \leq A \& \varepsilon<\varepsilon_{0} \\
& \left.\Rightarrow \forall t \in[0, s / \omega(\varepsilon)]\left\|x^{(f, G)}\left(t,\left(x_{0}, y_{0}\right), \varepsilon\right)\right\| \leq B\right)
\end{aligned}
$$

This is the usual definition of being $\omega$-UIB when $\varepsilon \rightarrow 0$.

\section{Nonstandard proofs of standard theorems}

In this section we show how to deduce the internal theorems 2.3, 5.6 and 5.9 from their external translations 2.2, 4.8 and 4.13. First we need the following external characterization of PSGAS.

Proposition D.1 Assume that $F$ is standard. The origin of (18) is PSGAS when $\varepsilon \rightarrow 0$ if and only if it is $\mathrm{S}-G A S$ for all positive infinitesimal $\varepsilon$.

Proof. To say that the origin of (18) is S-GAS for all positive infinitesimal $\varepsilon$ is the same as saying that

$$
\forall \varepsilon \forall z_{0} \forall t\left(z_{0} \text { limited \& } t \simeq+\infty \& \varepsilon \simeq 0 \Rightarrow z^{F}\left(t, z_{0}, \varepsilon\right) \simeq 0\right) .
$$

Using (36) this formula becomes

$$
\forall \varepsilon \forall z_{0} \forall t\left(\exists^{\text {st }} A \forall^{\text {st }} T \forall^{\text {st }} \varepsilon_{0} P \Rightarrow \forall^{\text {st }} \delta>0 Q\right)
$$

where $P$ is the internal formula $\left\|z_{0}\right\| \leq A \& t>T \& \varepsilon<\varepsilon_{0}$ and $Q$ is the internal formula $\left\|z^{F}\left(t, z_{0}, \varepsilon\right)\right\|<\delta$. Here, $F$ is a standard parameter. By (34), this is equivalent to

$$
\forall A \forall \delta>0 \exists^{\text {fin }} \varepsilon_{0}^{\prime} \exists^{\text {fin }} T^{\prime} \forall \varepsilon \forall z_{0} \forall t\left(\forall T \in T^{\prime} \forall \varepsilon_{0} \in \varepsilon_{0}^{\prime} P \Rightarrow Q\right) .
$$

For $T^{\prime}$ a finite set, $\forall T \in T^{\prime} t>T$ is the same as $t>T$ for $T=\max T^{\prime}$. For $\varepsilon_{0}^{\prime}$ a finite set, $\forall \varepsilon_{0} \in \varepsilon_{0}^{\prime} \varepsilon<\varepsilon_{0}$ is the same as $\varepsilon<\varepsilon_{0}$ for $\varepsilon_{0}=\min \varepsilon_{0}^{\prime}$ and so our formula is equivalent to

$$
\forall A \forall \delta>0 \exists \varepsilon_{0} \exists T \forall \varepsilon \forall z_{0} \forall t\left(\left\|z_{0}\right\| \leq A \& T t>T \& \varepsilon<\varepsilon_{0} \Rightarrow\left\|z^{F}\left(t, z_{0}, \varepsilon\right)\right\|<\delta\right) .
$$

This is the usual definition of the convergence of $z^{F}\left(t, z, \varepsilon_{0}\right)$ to 0 when $t \rightarrow+\infty$ and $\varepsilon \rightarrow 0$, the convergence being uniform with respect to the initial condition $z_{0}$ for $\left\|z_{0}\right\|<$ $A$. Thus the origin is PSGAS when $\varepsilon \rightarrow 0$.

Proof. [ of Theorem 2.3] By the transfer principle, we assume that $f$ and $G$ are standard. Let $\varepsilon \simeq 0$. By Theorem 2.2, the origin of system (6) is S-GAS. By Proposition D.1, the origin of (6) is PSGAS when $\varepsilon \rightarrow 0$. 
Proof. [of Theorem 5.6] By the transfer principle, we assume that $f$ and $G$ are standard functions. Assume that H6 holds. Since the origin of $\dot{y}=G(y, \varepsilon)$ is IS when $\varepsilon \rightarrow 0$, by Proposition C.1, it is IS for all $\varepsilon \simeq 0$. Since (1) is UIB when $\varepsilon \rightarrow 0$, by Proposition C.2, it is UIB for all $\varepsilon \simeq 0$. Hence H4 holds. By Theorem 4.8, the origin of (1) is S-GAS for all $\varepsilon \simeq 0$. By Proposition D.1, the origin of (1) is PSGAS when $\varepsilon \rightarrow 0$.

Proof. [of Theorem 5.9] By the transfer principle we assume that $f$ and $G$ are standard functions. Assume that $\mathrm{H} 7$ holds. By the transfer principle, there exists a standard positive function $\omega$ such that $\mathrm{H} 7$ holds. We have $\omega(\varepsilon) \simeq+\infty$ for all $\varepsilon \simeq 0$. Since the origin of $\dot{y}=G(y, \varepsilon)$ is $\omega$-IS when $\varepsilon \rightarrow 0$, by Proposition C.3, it is $\omega(\varepsilon)$-IS for all $\varepsilon \simeq 0$. Since (1) is $\omega$-UIB when $\varepsilon \rightarrow 0$, by Proposition C.4, it is $\omega(\varepsilon)$-UIB for $\varepsilon \simeq 0$. Thus H5 holds. By Theorem 4.13, the origin of (1) is S-GAS for all $\varepsilon \simeq 0$. By Proposition D.1, the origin of (1) is PSGAS when $\varepsilon \rightarrow 0$.

\section{E. NSA and differential equations}

G. Reeb was certainly the first to see all the benefit which NSA could bring to the drafting in the field of ordinary differential equations where the geometrical arguments are not allways simple to formalize. He pushed at the end of the seventies some young researchers of Mulhouse, Oran, Strasbourg and Tlemcen to be interested in the equation of Van der Pol via NSA. In the France of mathematics which hardly began to conceive that there were other pure mathematics than Bourbaki's ones and other applied mathematics than that of the digital simulation of partial differential equations, to push mathematicians to be interested in a very small equation that only the electronics specialists of the schools of engineers taught, was resolutely provocative. G. Reeb did not doubt that on this old subject a new glance would not fail to be fertile. It is what occurred with the discovery of the phenomenon called canard (or duck) phenomenon, i.e. of the importance of certain special solutions in the description of phase portrait of certain one parameter families of differential equations [1]. Our treatment of the peaking phenomenon very clearly claims the philosophy inaugurated in this article. NSA made many other intrusions in the theory of differential equations like, for example, the stroboscopic method, which is the external vision of the classical averaging methods [5, 28], the theory of differential equations with discontinuous right hand sides [18], the theory of rivers [9], which does not have yet a classical equivalent, and the consideration of the complex slow-fast differential equations [4]. We return to [7] and its bibliography for a rather complete vision of the subject.

\section{F. References}

[1] Benoit B. , Callot J.L, Diener F. , Diener M.. "Chasse au canard”. Collectanea Mathematica, vol. 31, num. 1-3 (1981) 37-119.

[2] VAN DEN Berg IP., "Nonstandard Asymptotic Analysis", Springer Lecture Notes in Math., vol. $1249,1987$.

[3] BYRNES C.I. , ISIDORI A., "Bifurcation analysis of the zero dynamics and the practical stabilization of nonlinear minimum-phase systems", Asian Journal of Control, 4(2) (2002), 171-185. 
[4] Callot JL.. "Champs lent-rapides complexes à une dimension lente", Ann. Scient. Ec. Norm. Sup, vol. 26, num. 4, (1993) 149-176.

[5] Callot JL., SaRi T., "Stroboscopie infinitésimale et moyennisation dans les systèmes d'équations différentielles à solution rapidement oscillantes", in Outils et Modèles Mathématiques pour l'Automatique, l'Analyse de Systèmes et le Traitement du Signal, I. D. Landau (editor), Volume 3. Edition du CNRS, Paris, 1983., pages 345-353.

[6] Canalis-Durand M., Yalo P., "Robustesse des systèmes linéaires bouclés aux perturbations non linéaires", in Analysis and Optimization of systems, A. Bensoussan and J. L. Lions (editors), Springer Lecture Notes in Control and Information Sciences, , vol. 111 (1988) 2782293.

[7] Diener F., Diener M., "Nonstandard Analysis in Practice", Universitext, Springer Verlag, 1995.

[8] Diener F., Reeb G., “Analyse non standard”, Hermann, Paris, 1985.

[9] Diener M., ReEB G., "Champs polynômiaux: nouvelles trajectoires remarquables", Bull. Soc.Math. Belgique, 38 (1987) 131-150.

[10] Diener M., Wallet G. (Editeurs), “ Mathématiques finitaires et analyse non standard”, Publication mathématique de l'Université de Paris 7, vol. 31, num. 1-2, 1989.

[11] Gauthier JP., KUPKA IAK., "Deterministic Observation Theory and Applications", Cambridge University Press, 2001.

[12] HAHN W., "Stability of Motion”, Springer Verlag, Berlin, 1967.

[13] Hoppensteadt F., "Singular perturbations on the infinite interval", Trans. Amer. Math. Soc., vol. 123 (1966) 521-535.

[14] Khalil HK., "Nonlinear systems”, Prentice Hall, 1996.

[15] Kokotovic P.V., Marino R.. "On vanishing stability regions in non-linear systems with high gain feedback”, IEEE. Trans. Autom. Control., AC-31(10), 1986.

[16] Lobry C., SARI T., "The Peaking Phenomenon and Singular Perturbations : an extension of On Tykhonov's theorem", Rapport de recherche INRIA, vol. 4051, (2000), 32 pages. http://www .inria.fr/rrrt/rr-4051.html

[17] LOBRY C., SARI T., "Singular perturbation methods in control theory", in Contrôle non linéaire et Applications, Travaux en Cours, vol. 64, Hermann, Paris (2005) , 155-182.

[18] LOBRY C., SARI T., "Equations différentielles à second membres discontinus", in Contrôle non linéaire et Applications, Travaux en Cours , vol. 64, Hermann, Paris (2005), 237-266.

[19] Lobry C., SARI T., "Nonstandard Analysis and representation of reality", International J. Control, Special Issue Dedicated to Professor Michel Fliess, vol. 81, num. 3 (2008) 519-536.

[20] Lobry C., SARI T., TouhAMi S., "On Tykhonov's theorem for convergence of solutions of slow and fast systems", Electronic J. Differential Equat., vol. 1998, num. 19 (1998) 1-22.

[21] Lobry C., SARi T., Touhami S., "Fast and slow feedbacks in systems theory,", J. Biol. Systems, vol. 7, num. 3 (1999) 1-25.

[22] Luenberger DG., "Observers for multivariable systems", IEEE Trans. Aut. Control, vol. AC, num. 11 (1966) 190-197.

[23] Lutz R., GozE M., "Nonstandard Analysis: a practical guide with applications", Springer Lecture Notes in Math., vol. 881, 1982.

[24] Lutz R., SARI T., "Applications of Nonstandard Analysis in boundary value problems in singular perturbation theory",in Theory and Applications of singular peturbations (Oberwolfach 1981) Springer Lecture Notes in Math., vol. 942 (1982) 113-135.

[25] Nelson E., "Internal Set Theory : a new approach to nonstandard analysis", Bull. Amer. Math. Soc., vol. 83 (1977) 1165-1198. 
[26] Robinson A., "Nonstandard Analysis”, merican Elsevier, New York, 1974.

[27] Rouche N., Habets P., LALoy M., "Stability Theory by Liapunov's Direct Method", Applied Mathematical Sciences, vol. 22, Springer Verlag, Berlin-New York, 1977.

[28] SARI T., "Averaging for ordinary differential equation and functional differential equations", In The Strength of Nonstandard Analysis, I. van den Berg, V. Neves (editors). Springer-Verlag, Wien, 2007, pages 286-305.

[29] Sussmann HJ., Kокотоvic PK., "The peaking phenomenon and the global stabilization of nonlinear systems", IEEE Trans. Aut. Control, vol. 36, num. 4 (1991) 424-440.

[30] Zvonkin AK., Shubin MA., "Nonstandard Analysis and Singular Perturbations of Ordinary Differential Equations,", Uspekhi Mat. Nauk, vol. 39 (1984) 77-127. 